\title{
Permanent magnet helicon source for ion propulsion
}

\author{
Francis F. Chen
}

Electrical Engineering Department, University of California, Los Angeles, California 900951594

\begin{abstract}
Helicon sources have been proposed by at least two groups for generating ions for space propulsion: the HDLT concept at the Australian National University (ANU), and the VASIMR concept at the Johnson Space Center in Houston. These sources normally require a large electromagnet and power supply to produce the magnetic field. At this stage of research, emphasis has been on the plasma density and ion current that can be produced, but not much on the weight, size, impulse, and gas efficiency of the thruster. This paper concerns the source itself and shows that great savings in size and weight can be obtained by using specially designed permanent magnets (PMs). This PM helicon design, originally developed for plasma processing of large substrates, is extended here for ion thrusters of both the HDLT and VASIMR types. Measured downstream densities are of order $10^{12} \mathrm{~cm}^{-3}$, which should yield much higher ion currents than reported so far. The design principles have been checked experimentally, showing that the predictions of the theory and computations are reliable. The details of two new designs are given here to serve as examples to stimulate further research on the use of such sources as thrusters.
\end{abstract}

\section{Introduction}

Helicon sources fall into the category of Inductively Coupled Plasmas (ICPs), which use radiofrequency $(\mathrm{RF})$ antennas to create plasma without internal electrodes. Since they are related to whistler waves, helicons exist only in a steady (DC) magnetic field ( $\equiv \mathbf{B}_{0}$ ). Sources based on helicon waves have been found to produce 3-10 times higher plasma density $(\equiv n)$ than field-free ICPs in manufacturing applications. In 2003, supersonic ions from helicon sources were found by S. A. Cohen et al. ${ }^{1}$ and by C. Charles and R.W. Boswell, who discovered that a current-free double layer (DL) occurs downstream of a helicon source expanding into a diverging magnetic field ${ }^{2,3}$, and that the ion beam is accelerated in the thin, collisionless layer ${ }^{4}$. This concept was named the Helicon Double Layer Thruster (HDLT). In addition to the potential jump in the DL, Ref. 4 contains detailed measurements of the ion velocity distribution using a retarding-field energy analyzer at typically $250 \mathrm{~W}$ of RF power and $0.35 \mathrm{mT}$ Torr of Ar. These ion energy peaks at the $29 \mathrm{eV}$ plasma potential and at $47 \mathrm{eV}$ behind the DL. The latter is supersonic at $2.1 c_{\mathrm{s}}$, where $c_{\mathrm{s}}$ is the ion sound velocity. The DL has been shown pictorially by Charles ${ }^{5,6}$. These results were confirmed by Keesee et al. ${ }^{7}$ using laser-induced fluorescence and by Sun et al. ${ }^{8}$ on the helicon machine at West Virginia University. Further confirmation was given by the PICMCC simulations of Meige et al. ${ }^{9,10}$ A machine ${ }^{11}$ constructed at the Ecole Polytechnique in France to reproduce the HDLT experiment fully confirms and extends the ANU results. All experiments on the HDLT were recently reviewed by Charles ${ }^{12}$. The specific impulse and thrust of the HDLT were estimated to be low compared with conventional thrusters, but the authors anticipated that these could be increased with improvements in source efficiency. This paper is intended to provide such improvements. 
That a single layer should form in an expanding B-field was shown by $\mathrm{Chen}^{13}$ to be a result of the Bohm sheath criterion, which is normally applied to the condition at a wall or floating probe that will maintain quasineutrality. In this instance, the ion acceleration to the Bohm velocity $c_{\mathrm{s}}$ that normally occurs in a pre-sheath occurs in an expanding B-field, since perpendicular ion energy is converted into parallel energy. In the absence of a wall, the single sheath has to turn into a double sheath so that the potential will flatten out, else the ions will be accelerated indefinitely without an additional source of energy. Chen ${ }^{13}$ predicted that the DL should occur where the B-field has decreased by $e^{-1 / 2}=0.61$. A measurement of the DL position by Sutherland et al. ${ }^{14}$ verified this prediction to within $3 \%$. In varying the B-field, Charles ${ }^{15}$ found that the DL depended on the field near the back plate. This effect is probably related to wave reflection at the back plate, an effect used in source optimization in this paper. Finally, Gesto et al. ${ }^{16}$ have calculated the surface where ion detachment from the B-field occurs. This is deemed important in hydrogen plasmas (next paragraph) where high beta is necessary for detachment, but it is probably not important for the argon plasmas used so far in DL experiments, since the ion Larmor radii are larger than the chamber radii.

The second large group using a helicon source for ion propulsion is that of F.R. ChangDiaz in the Variable Specific Impulse Magnetohydrodynamic Rocket (VASIMR) project ${ }^{17}$. In this concept, hydrogen or deuterium ions are ejected from a helicon source immersed in $\mathbf{B}_{0}$. The charge-neutralized ion beam is then compressed when it enters a stronger magnetic field and through a small aperture used for differential pumping. In the low-pressure, high B-field region, the ions are heated by ion cyclotron resonance (ICRH); and the subsequent expansion into a weak B-field converts the ions' perpendicular energy into parallel energy. Finally, magnetic nozzles ${ }^{18}$ are used to shape the exiting beam for maximum thrust. In the helicon section, the requisite B-field cannot be created with small solenoids because the field lines curve back, preventing the plasma from propagating downstream unless its beta is large enough to break through the field lines.

The size and complexity of the helicon source in either HDLT or VASIMR can be greatly reduced by employing permanent magnets to create the B-field. Previous attempts to do this by placing the plasma tube inside ring magnets suffered from the same deficiency as small solenoids: the field lines curved backward upon leaving the tube, preventing ejection of the plasma. However, annular magnets have a stagnation point beyond which the field lines extend toward infinity. By placing the plasma tube in the weaker field beyond the stagnation point, plasma can be ejected from the source even at low beta. Furthermore, the permanent magnets can be relatively small and require no power supply. This concept has been proven experimentally ${ }^{19,20}$. The original design in Ref. 19 was for large-area plasma processing but can be adopted without change as an upgrade of the source in the HDLT. This is done in Sec. III.

Experiments on the VASIMR engine have been carried out at the Johnson Space Center in Houston using light gases in a 9-cm diam helicon source ${ }^{21}$. The use of gases lighter than $\mathrm{Li}$ was dictated by the ICRH section, where heavier gases would require overly high B-fields to keep the ion Larmor radii small. The helicon section itself has been studied at the Oak Ridge National Laboratory ${ }^{22}$ with deuterium in a 5-cm diam tube. Supporting experiments on a highpower helicon discharge in argon, also with an inner diameter of order $5 \mathrm{~cm}$, were done at the University of Washington ${ }^{18,23}$. To correlate with these existing experiments, the present calculations were done for both 9- and 5-cm diameter plasmas in Secs. IV and V, respectively.

Although enhancement of ICPs with PMs has been investigated by a number of authors, very few papers report on the use of PMs for helicon discharges ${ }^{24,25}$. In particular, there are no papers other than Ref. 19 on the use of the external field of annular magnets. 


\section{Methodology}

This design of an optimized PM helicon source relies on two innovations: 1) the low-field peak and 2) the HELIC code. In its simplest form, the dispersion relation for a helicon wave of frequency $\omega / 2 \pi$ in a long circular cylinder can be written

$$
k_{r}=\frac{\omega}{k_{z}} \frac{n e \mu_{0}}{B}, \text { so that } \frac{1}{a} \propto \frac{\omega}{k} \frac{n}{B}
$$

where $k_{\mathrm{r}}$ is an effective radial wavenumber (inversely proportional to the plasma radius $a$ ), $k_{z}$ (or $k$ ) is the axial wavenumber, and $n$ and $B$ the plasma density and RF B-field. In a uniform plasma, $k_{\mathrm{r}}$ is given by a Bessel function root, and $n$ is a constant; but radial non-uniformity requires computation. Nonetheless, the proportionalities in Eq. (1) allow us to predict the direction to go during optimization. Equation (1) shows that $n$ should increase linearly with $B$, but it was found that $n$ has a small peak at low B-fields. This low-field peak was subsequently explained ${ }^{26,27}$ by constructive interference of the helicon wave reflected from the endplate near the antenna. It occurs with bi-directional antennas, such as the Nagoya Type III and $m=0$ (azimuthally symmetric) antennas, but not with helical antennas, which excite $m=+1$ helicon waves in only one direction. Diverging (cusp) magnetic fields upstream of the antenna can also create a low-field peak by bringing the field lines against the sidewalls, which then act as an endplate $^{26}$. This effect was observed long $\operatorname{ago}^{28}$ and has been rediscovered in VASIMR experiments $^{21}$. By using the low-field peak, a given density can be produced with a smaller Bfield; and by using $m=0$ antennas, plasma wall losses under the antenna can be greatly reduced.

The HELIC code of D. Arnush ${ }^{29}$, based on analytic theory, made possible rapid scans of parameter space to find optimum absorption of RF power. A user-friendly version of this code for personal computers can be obtained from the author ${ }^{30}$. The program assumes a plasma of radius $a$, a thin antenna of radius $b$, and a ground plane at radius $c$, as shown in Fig. 1 . The confining cylinder can be infinitely long or bounded by insulating or conducting endplates
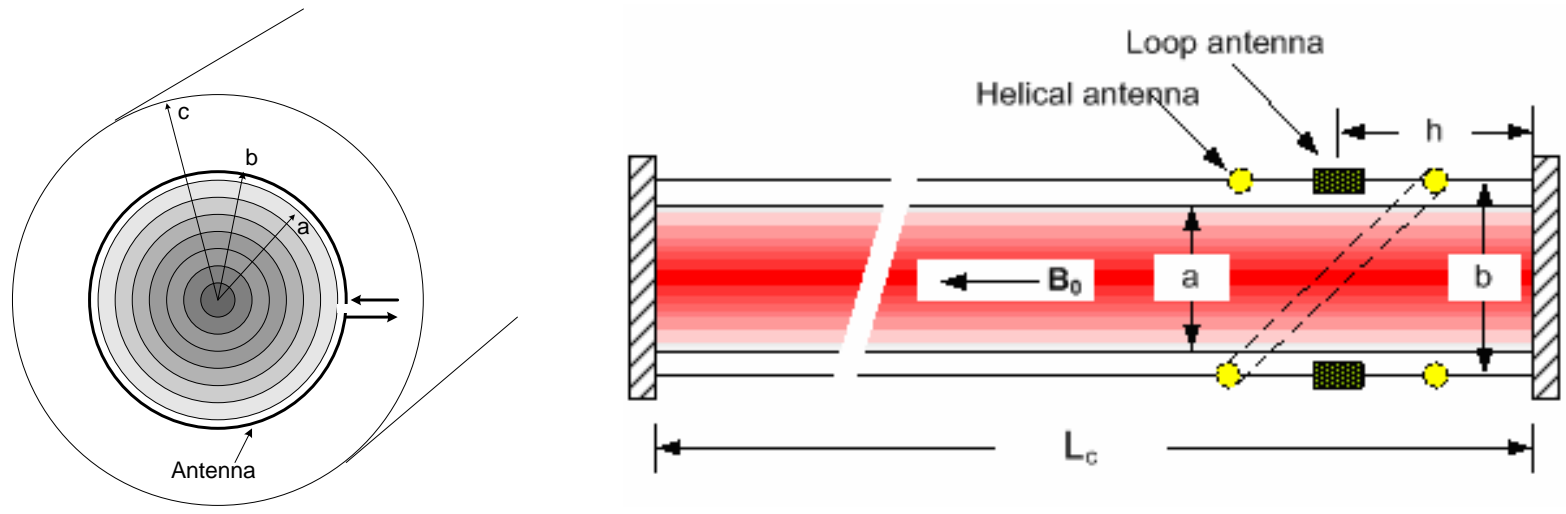

Fig. 1. Geometry of the HELIC program

separated by the cylinder length $L_{\mathrm{c}}$. The antenna can be any of the common types, or a new one can be specified by its Fourier transform. The midplane of the antenna can be set at an adjustable distance $h$ from one endplate. The main constraint which allows for rapid computation is that the equilibrium density $n$ and magnetic field $\mathbf{B}_{0}$ must be uniform in the axial direction $z$. To simulate ejection from a small discharge tube, $L_{\mathrm{c}}$ in this paper is set at a large value of 2 meters, while $h$ is of the order of centimeters. The radial profiles of density, electron temperature $T_{\mathrm{e}}$, and neutral pressure $p_{0}$ can also be specified. We use the convenient parametrization of Eq. (2) to vary $n(r)$. Here three arbitrary numbers $s, t$, and $w$ (or $f_{\mathrm{a}}$ ) are used 
to match an arbitrary, smooth profile between $r=0$ (where $n=n_{0}$ ) and $r=a$ (where $n=n_{\mathrm{a}}$ ). Also, $f_{\mathrm{a}}$ is the fractional density at $r=a$, and $w$ is the "width" of the profile and is directly related to $f_{\mathrm{a}}$. Electrons are assumed to be Maxwellian. Consequently, $T_{\mathrm{e}}$ affects mainly the plasma potential, which does not enter the design, though it affects the ejected ion energy. The collision frequency does depend on $T_{\mathrm{e}}$, but in high-power helicon discharges $T_{\mathrm{e}}$ usually lies between 3 and $4.5 \mathrm{eV}$. The collision frequencies of a few gases are built into HELIC, but an adjustable collision factor can accommodate other gases.

$$
\frac{n}{n_{0}}=\left[1-\left(\frac{r}{w}\right)^{s}\right]^{t}, \quad \frac{n_{a}}{n_{0}}=\left[1-\left(\frac{a}{w}\right)^{s}\right]^{t} \equiv f_{a}
$$

Once the geometry, $n(r), \mathbf{B}_{0}, p_{0}, \omega$, antenna type, and gas have been specified, the program solves a fourth-order differential equation for each of several hundred values of $k$ to obtain the radial and axial profiles of the RF current and electric and magnetic fields, as well as the profiles of the energy absorbed. The latter are integrated to obtain the plasma loading resistance $R_{\mathrm{p}}$, our principal result. The absorption of RF energy is dominated by mode conversion to heavily damped Trivelpiece-Gould (TG) modes $^{31}$ at the radial surface, and this Shamrai $^{32}$ effect is fully accounted for in HELIC. Electromagnetic radiation, ion mass effects (such as lower-hybrid resonance), and Landau damping are included, but none of these effects is important in the parameter regime of this paper. Helicon discharges with endplates are known to exhibit axial resonances ${ }^{33,34}$. The low-field peak treated here is a special case in which there is only one endplate. For reasons which will become clear, our design uses $m=0$ antennas. The behavior of such antennas has also been studied by numerous authors ${ }^{35-37}$.

The HELIC program can also scan ranges of $n$ or $B_{0}$, or both at the same time, with either linear or logarithmic spacing of the points. Data for $R_{\mathrm{p}}$ vs. $n$ for various $B_{0}$ such as the ones shown below typically take $2-3$ hours to generate on a $400-\mathrm{MHz}$ PC. Programming to take advantage of faster CPUs was not available when HELIC was written. The code has not been calibrated in a dedicated experiment, but its predictions have been verified in at least two cases. Blackwell et al. ${ }^{38}$ detected the Trivelpiece-Gould modes directly, in agreement with HELIC calculations, by measuring the RF current. Chen and Torreblanca ${ }^{39}$ found that the absolute value of $R_{\mathrm{p}}$ from HELIC agreed within experimental error with the value obtained from the jump into the helicon mode. The fact that the assumed uniform B-field disagrees with the actual diverging B-field in ejection experiments is not important because little ionization or wave reflection occurs outside the source. This was proved in Ref. [19] and subsequent experiments, in which the optimum conditions agreed with those predicted.

\section{Design of an HDLT source}

Medusa 2 is an 8-tube array of small helicon sources at UCLA to test production of a large-area, high-density, uniform plasma with multiple sources. A single source of the same design can be used for the low-power applications of the HDLT without modification. The field of a stack of annular permanent magnets is shown in Fig. 2. Note that a plasma created inside the strong field region cannot be ejected because of the field lines run into the wall. However, there is a stagnation point at which the B-field reverses sign. A plasma placed in the field beyond the stagnation point will be in a slowly diverging field. Figure 3 shows a proof-ofconcept experiment in which it was shown that a helicon discharge could be produced in the farfield. The magnetic field can be varied by changing the magnet height D. Figure 4 shows radial profiles taken with fully RF-compensated Langmuir probes at an RF power $P_{\mathrm{rf}}=500 \mathrm{~W}$ at 13.56 
$\mathrm{MHz}$ and $\mathrm{D}=15 \mathrm{~cm}$. The probe positions are $\mathrm{Z1}=7.4 \mathrm{~cm}$ and $\mathrm{Z2}=17.6 \mathrm{~cm}$ below the tubeflange junction. Two pressures, 4 and $10 \mathrm{mTorr}$ of argon are shown. These are for plasma processing and are higher than would be used in a thruster. They are fill pressures, not the neutral pressure inside the tube during the discharge. The flattened profiles at $\mathrm{Z} 2$ are caused both by the B-field divergence and by diffusion at these pressures. The peak density at Z1 is 0.8 $\times 10^{12} \mathrm{~cm}^{-3}$.

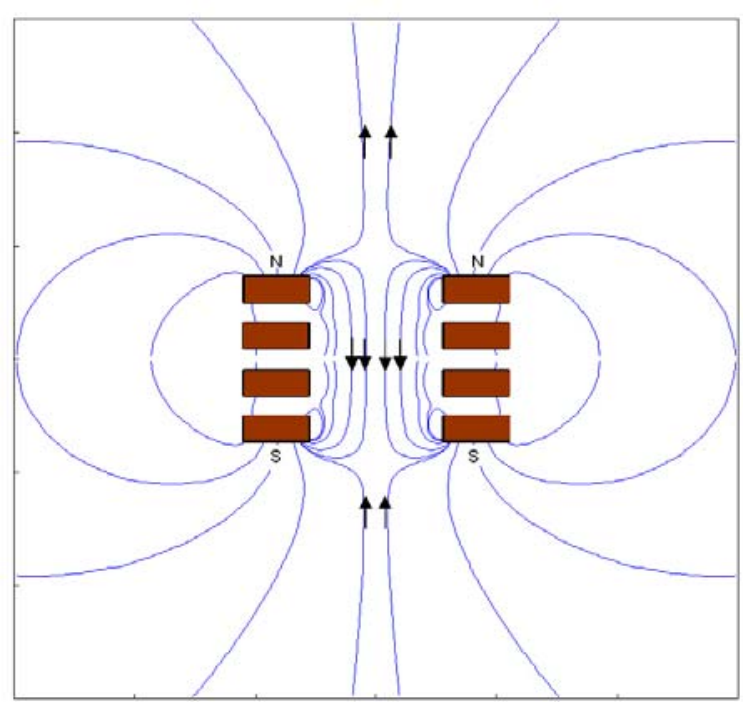

Fig. 2. Field lines of annular magnets.

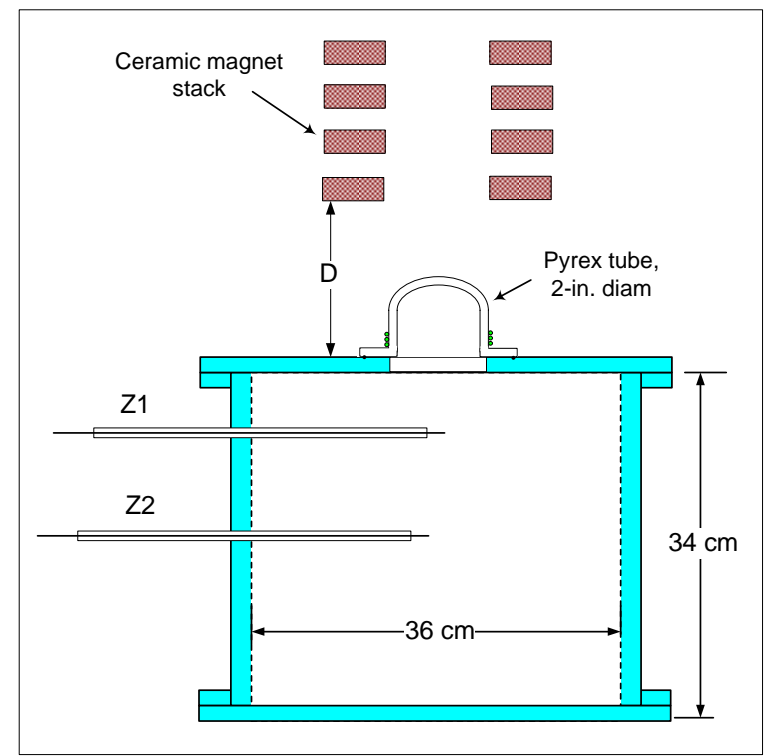

Fig. 3. Discharge tube in the far-field.

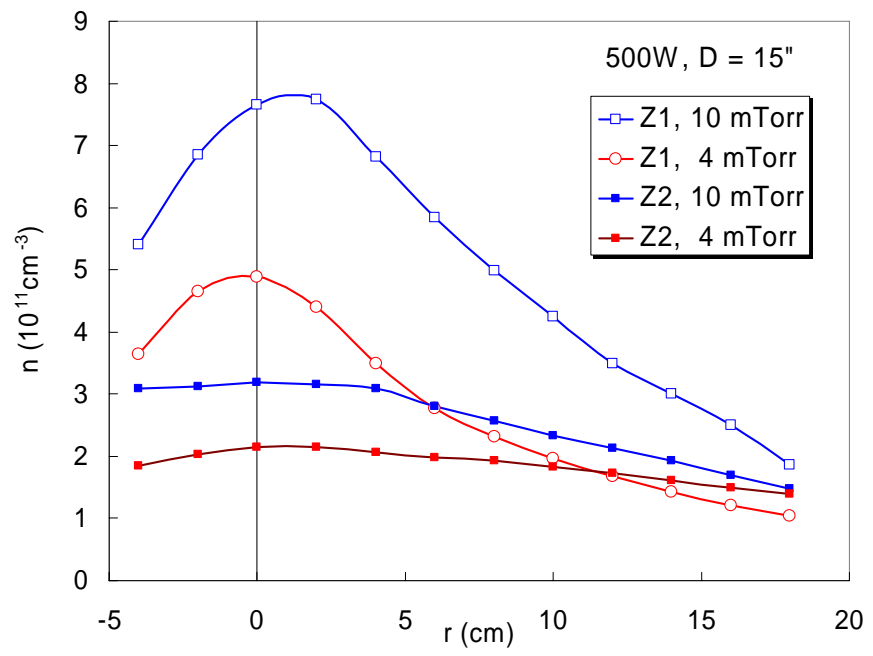

Fig. 4. Radial density profiles in Fig. 3 at two probe positions and two pressures.

The discharge tube and magnet were then optimized in the manner described in the next section. The details will be omitted for this case, since they are available elsewhere ${ }^{19,40}$. The tube, magnet, and antenna optimized for $13.56 \mathrm{MHz}$ are shown in Fig. 5. The tube is quartz or alumina, and the top plate (without gas feed) can be a standard $50-\mathrm{mm}$ diam aluminum cover with O-ring mount. The NeFeB magnet is made in two pieces, each with $7.6 \mathrm{~cm}$ I.D., $12.7 \mathrm{~cm}$ O.D., and $1 \mathrm{~cm}$ thickness. They are supported by an aluminum sheet. The B-field at the antenna 
position is about $80 \mathrm{G}$ at the optimal magnet distance $\mathrm{D}$. The antenna consists of three turns of 3.2-mm diam water-cooled copper tubing. Engineering problems with the RF system, connections, etc. will be discussed in Sec. IV. The density produced by this source is shown in Fig. 6.

Since this source was made for plasma processing, the pressure was higher than for thruster applications; and no measurements of ion flux or energy downstream were made. Diagnostics for detection of DLs are not available. However, should DLs occur with this source, the available thrust should be much higher than in HDLT experiments so far. Walker et al. ${ }^{11}$ measured a plasma density of $3 \times 10^{9} \mathrm{~cm}^{-3}$ at $13 \mathrm{~cm}$ from the end of the antenna with $500 \mathrm{~W}$ of RF power and an argon pressure $p_{0}=0.2$ mTorr. From Fig. 6 we obtain $n \approx 5 \times 10^{11} \mathrm{~cm}^{-3}$ at a position between $\mathrm{Z1}=7.4 \mathrm{~cm}$ and $\mathrm{Z} 2=17.6 \mathrm{~cm}$ from the antenna at $p_{0}=10 \mathrm{mTorr}$ and the same $P_{\text {rf. }}$ Thus, the density is $\approx 170$ times higher at a pressure 50 times higher. At $500 \mathrm{~W}$ the pressure within the small tube volume should be lower than the fill pressure, so the gain of approximately a factor of 3 may actually be higher. Mainly, the heavy magnet coils and DC power supply are avoided. Since the endplate of the PM source can be biased to an arbitrary potential, the plasma potential in the source can be made positive to increase the ion beam velocity. Perhaps this can be done to a limited extent without incurring spacecraft charging. Should electron neutralization of the ion beam be necessary, a very small PM helicon source can be designed for that purpose.

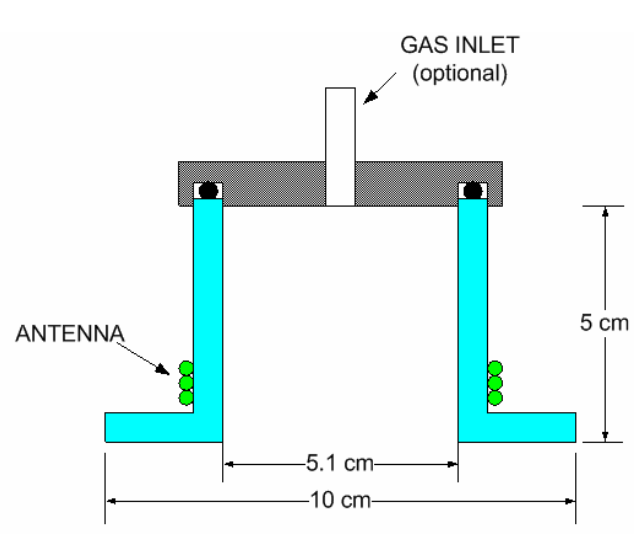

(a)

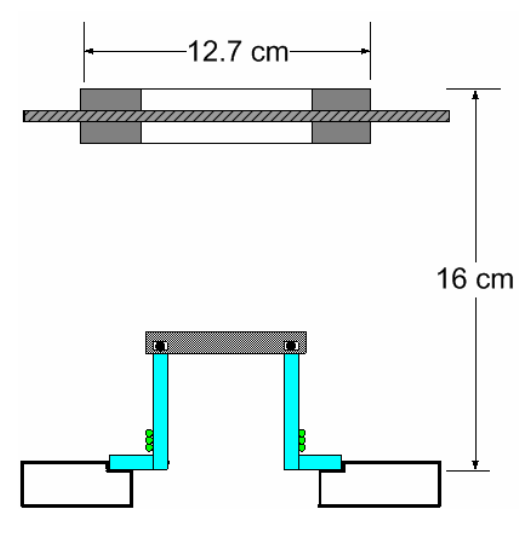

(b)

Fig. 5. (a) Optimized 2-in. diam discharge tube; (b) relative positions of tube and magnet.

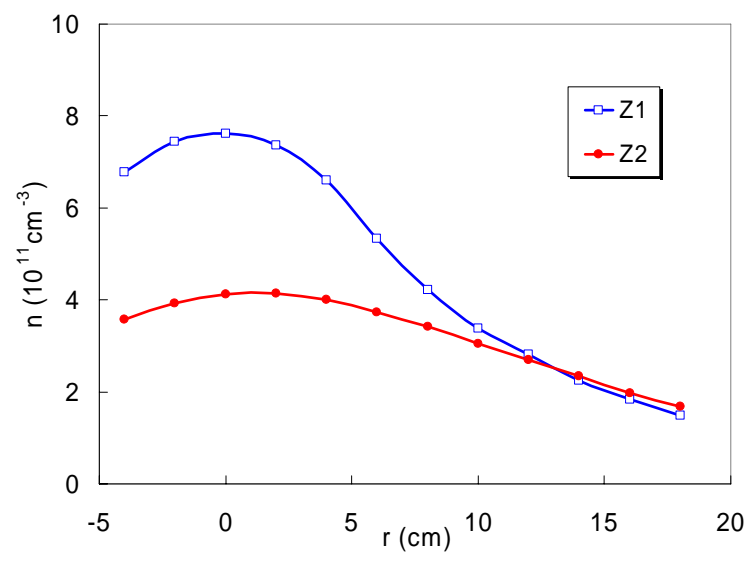

Fig. 6. Density profiles measured at ports Z1 and Z2 in the source of Fig. 5 with $P_{\mathrm{rf}}=500 \mathrm{~W}$ and $10 \mathrm{mTorr}$ of Ar. 


\section{Design of a 9-cm diameter VASIMR source}

Since the VASIMR is an approach in which the size and weight of the source and its power supplies are not considered in the attempt to create maximum thrust, we have pushed the PM helicon source design to high RF powers. The $9 \mathrm{~cm}$ diam was chosen to match the tube diameter currently used in VASIMR experiments.

The rate of deposition of RF energy into a plasma depends on the loading resistance $R_{\mathrm{p}}$ at the output of the matching circuit. Since there are unavoidable losses in the RF circuitry, the name of the game is to make $R_{\mathrm{p}}$ much larger than the effective circuit resistance $R_{\mathrm{c}}$. In small discharge tubes, $R_{\mathrm{p}}$ tends to be too low $(<1 \Omega)$ unless one makes use of the low-field peak. The power actually reaching the plasma, $P_{\text {in }}$, is given by

$$
P_{\text {in }}=P_{r f} \frac{R_{p}}{R_{p}+R_{c}},
$$

where $P_{\mathrm{rf}}$ is the power applied from the RF generator. Thus, efficient use of $P_{\mathrm{rf}}$ requires $R_{\mathrm{p}}>>$ $R_{\mathrm{c}}$. Computations of $R_{\mathrm{p}}$ are made for the plasma tube shown in Fig. 7. The short, stubby aspect ratio

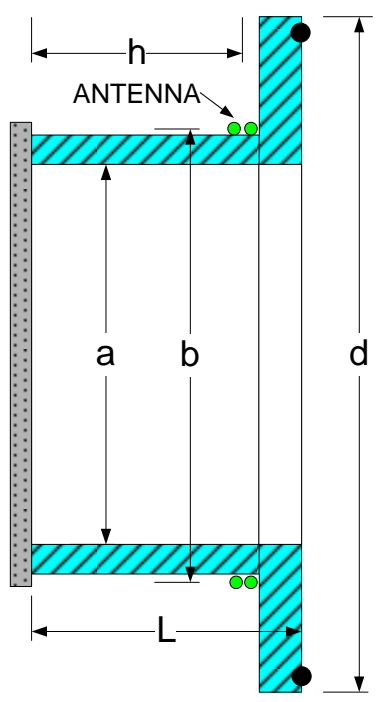

Fig. 7. Diagram of plasma tube being optimized. The antenna is an $m=0$ winding of $\mathrm{N}=2-3$ turns, with average radius $b$. Here $a$ is the i.d. of the tube, and $h$ is the distance from the endplate to the midplane of the antenna, as in Fig. 1. The skirt diameter $d$ is not computed but has to be large enough to minimize eddy currents in the mounting flange, and $L$ differs from $h$ by the skirt thickness and half the antenna length.

of this tube was found in previous work ${ }^{41}$ to give much higher downstream densities than other shapes. The tube length $L$ is short in order to take advantage of the low-field peak. As will be shown, most of the absorption (due to the TG mode) occurs near the boundary. Short $m=0$ antennas, therefore, do not suffer from the large wall losses that long antennas, such as helical or Nagoya antennas, incur. The antenna is located as close as possible to the exit aperture to maximize the ejected plasma. The wide "skirt" is needed to bring the antenna away from the mounting flange, which would have RF eddy currents if it is conducting. For steady-state operation, the tube gets hot and should be made of alumina. A conducting top plate can be attached with a metal-to-ceramic seal. A domed alumina top can be made in one piece ${ }^{35}$, but a conducting top has advantages, as will be seen. 
Figure 8 shows HELIC computations of $R_{\mathrm{p}}$ vs. $n$ for three values of $B$, showing the lowfield peak. The peak moves to higher $n$ as $B$ is raised, in accord with Eq. (1). Since high densities $\geq 10^{13} \mathrm{~cm}^{-3}$ are desired here, B-fields of 400-600G are needed instead of the $60-100 \mathrm{G}$ used for plasma processing. In these calculations argon gas is assumed for convenience. Lighter gases with different damping rates would have a small effect on HELIC calculations, but the power required to achieve these densities would be much higher. On the other hand, xenon would yield much higher densities.

Figure 9 shows the $R_{\mathrm{p}}-n$ curves at 400 and $600 \mathrm{G}$ as the tube length $L$ (actually, $h$ ) is varied for a fixed tube diameter of $9 \mathrm{~cm}$. At $600 \mathrm{G}, R_{\mathrm{p}}$ peaks at $n=10^{13} \mathrm{~cm}^{-3}$ and has high $R_{\mathrm{p}}$ throughout the range $1-3 \times 10^{13} \mathrm{~cm}^{-3}$. At $400 \mathrm{G}$, the $L=4 \mathrm{~cm}$ tube peaks at $10^{13} \mathrm{~cm}^{-3}$, but the $L=$ $5 \mathrm{~cm}$ tube has the same $R_{\mathrm{p}}$ there. The 3-cm tube performs better at $n=2-3 \times 10^{13} \mathrm{~cm}^{-3}$, but has much lower $R_{\mathrm{p}}$ at $10^{13} \mathrm{~cm}^{-3}$ than the 4- or 5 - cm long tubes. We have therefore chosen $h=5 \mathrm{~cm}$, or $L \approx 6 \mathrm{~cm}$, as the optimum tube length for this diameter; this is the aspect ratio shown in Fig. 7 . The idea is to raise $R_{\mathrm{p}}$ above $1 \Omega$, preferably to several ohms, so that $R_{\mathrm{p}}>>R_{\mathrm{c}}$ is satisfied.

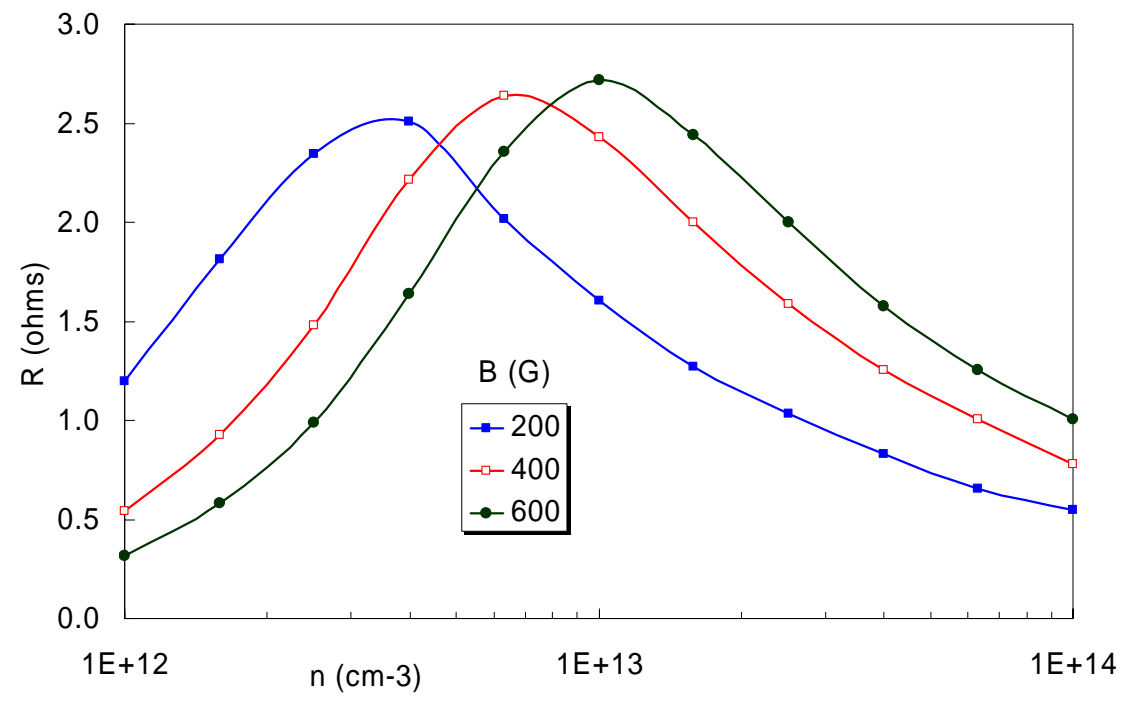

Fig. 8. Plasma resistance vs. density for a $9-\mathrm{cm}$ diam, $5 \mathrm{~cm}$ long source operating at $f=13.56 \mathrm{MHz}$. Unless otherwise specified, $p_{0}=10 \mathrm{mTorr}$ of argon, $K T_{\mathrm{e}}=3 \mathrm{eV}$, and a conducting endplate are assumed.

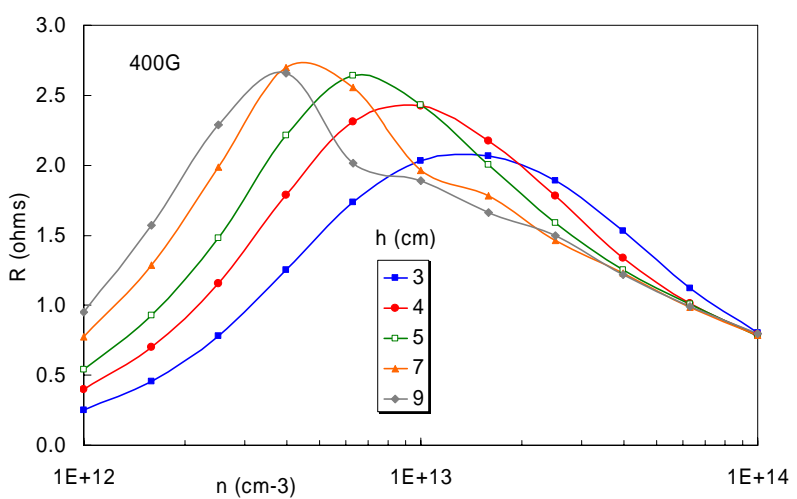

(a)

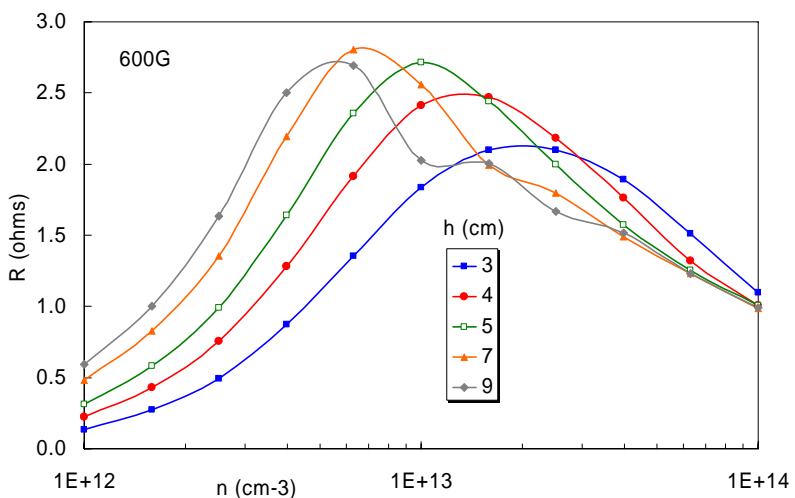

(b)

Fig. 9. $R_{\mathrm{p}}$ vs. $n$ at (a) 400 and (b) $600 \mathrm{G}$ as tube length is varied at $f=13.56 \mathrm{MHz}$. The order in the legend follows the order of the curves wherever possible. 
It should be noted that the HELIC program incurs errors at high fields and densities due to a problem that arises in all helicon computations. The two roots of the biquadratic dispersion equation then become mixtures of the helicon and TG modes, which are not clearly separated the way they are at low fields and densities. The solution requires finding a small difference between two large numbers. The program therefore switches to a high-field algorithm at some critical field which can be adjusted. The error is estimated in HELIC by calculating $R_{\mathrm{p}}$ by a) integrating the power deposition over the plasma volume, and b) calculating the phase shift in the antenna current and voltage. These two values of $R_{\mathrm{p}}$ normally differ by $1 \%$ or less, but at the $n$ and $B$ values of interest here the difference can be as large as $50 \%$. To be safe, we wish to achieve calculated $R_{\mathrm{p}}{ }^{\prime}$ s much larger than $1 \Omega$.

In Fig. 9, we note that the $h=4$ or $5 \mathrm{~cm}$ curve varies smoothly on both sides of its maximum, while at larger $h$ the curve has discontinuities in slope. This is an indication of multiple axial or radial modes. That this is the case is shown in Figs. 10 and 11 , in which the $k$ spectrum $P(k)$, the radial deposition profile $P(r)$, and the wave profile $\left|B_{\mathrm{z}}\right|(\mathrm{r})$ at $400 \mathrm{G}$ and $n=10^{13}$ $\mathrm{cm}^{-3}$ are compared for $h=4$ and $h=9 \mathrm{~cm}$. $P(k)$ is the relative power absorbed by the plasma at each Fourier component $k$ of the wave fields, where $k$ is $2 \pi$ divided by the parallel wavelength. $P(r)$ is the relative power deposited at each radius. At $h=4 \mathrm{~cm}$, there is one dominant mode whose reflection from the endplate interferes constructively with the forward wave. At $h=9 \mathrm{~cm}$, that mode is non-resonant, and there are two other competing modes at smaller $k$. The wave profiles in Fig. 11 are taken at the $z$ position of the antenna. The $h=4 \mathrm{~cm}$ case has a strong helicon component at the center, while the $h=9 \mathrm{~cm}$ case has smaller, higher-order radial modes. The absorption profile $P(r)$ is integrated over all $z$. It shows that the absorption is dominated by the TG peak at the edge, even though the wave amplitude is stronger at the center. This peculiarity of helicon discharges was not known in early papers on helicons. This is because the helicon mode's damping rate identically vanishes in first order ${ }^{29}$. Both tube lengths have essentially the same $P(r)$. Even though the highest $R_{\mathrm{p}}$ is obtained with pure modes, one can use the non-resonant case to advantage. If $R_{\mathrm{c}}$ is small enough so that $R_{\mathrm{p}}$ does not have to be maximized, a longer tube with multimodes can be used to obtain sufficiently large $R_{\mathrm{p}}$ over a wide density range.

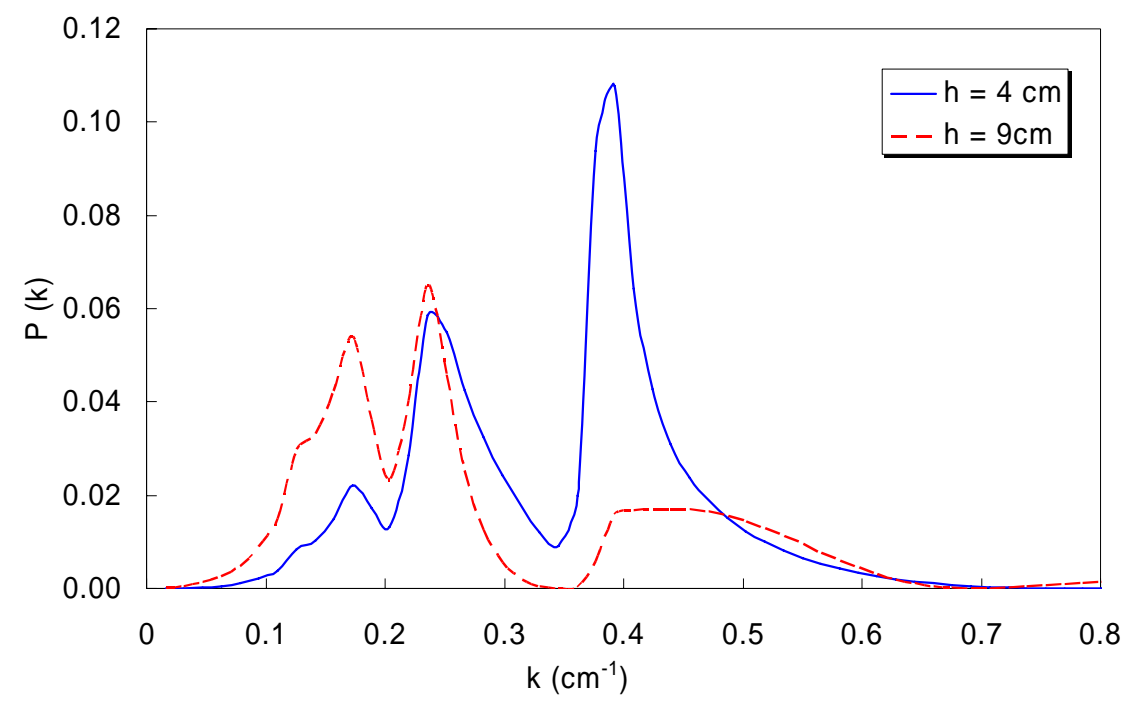

Fig. 10. The $k$ spectrum of power deposition for two tube lengths of a $9-\mathrm{cm}$ diam tube at $400 \mathrm{G}, 10^{13} \mathrm{~cm}^{-3}$, and 13.56 $\mathrm{MHz}$. 


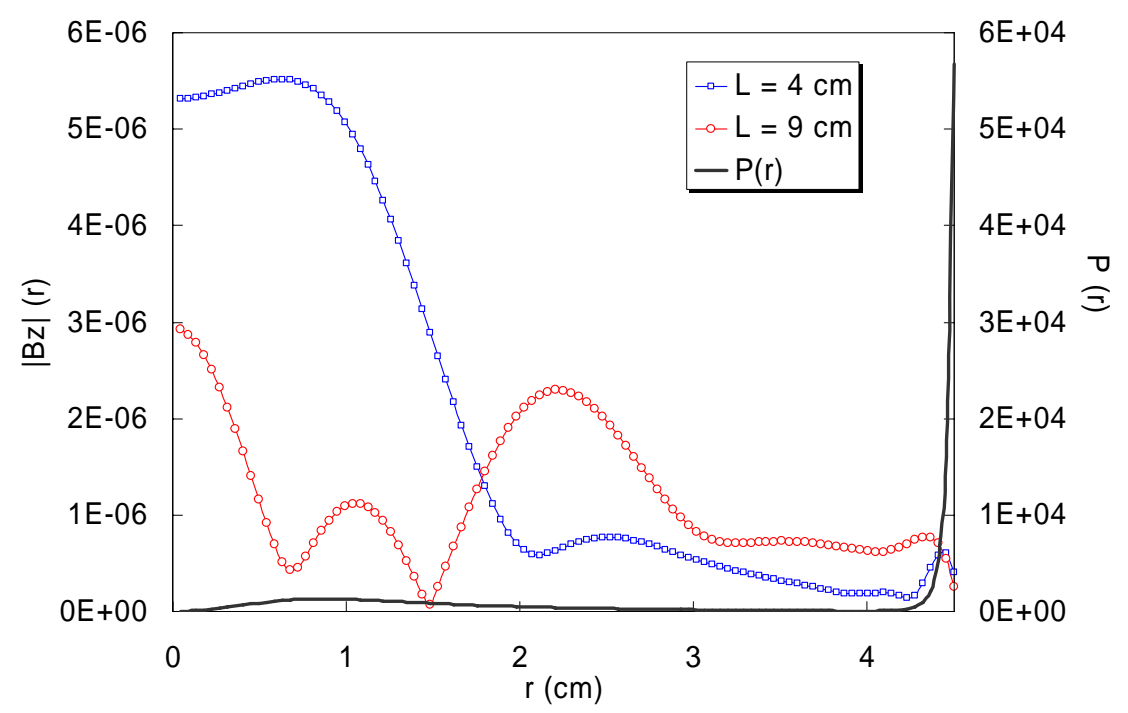

Fig. 11. Radial profiles of wave amplitude $\left|B_{z}\right|$ and power deposition $P(r)$ (r.h. scale) for the conditions of Fg. 5.

In Fig. 11, the TG peak in $P(r)$ is even more dominant than it appears because the factor $r$ in computing the volume is not included in $P(r)$. Since the plasma is mostly created at the edge, much of the density from a long antenna would be lost to the walls before reaching the exit aperture. At fixed $B$ in Fig. 9, the curves shift to higher $n$ when $k$ is increased by decreasing $h$, in agreement with Eq. (1). At fixed $k$, the curves shift to higher $n$ with increasing $B$, also in agreement with Eq. (1). This is true as long as there is one dominant mode.

The $R_{\mathrm{p}}$ results are sensitive to the assumed density profile. Figure 12 shows three profiles generated from Eq. (2) for different values of $s$, $t$, and $f_{\mathrm{a}}$. The corresponding $R_{\mathrm{p}}$ curves for $h=9$ $\mathrm{cm}$ and $400 \mathrm{G}$ are shown in Fig. 13. From these we see that $R_{\mathrm{p}}$ is larger for those curves (a and $\mathrm{b}$ ) with more density at the edge $\left(f_{\mathrm{a}}=0.3\right)$, where the TG mode is located. The flat profile in (c) suffers from having most of its density in the interior, where absorption is weak. A peaked profile is more likely at the high powers in this application because of neutral depletion, and profile (b) was used in subsequent calculations.

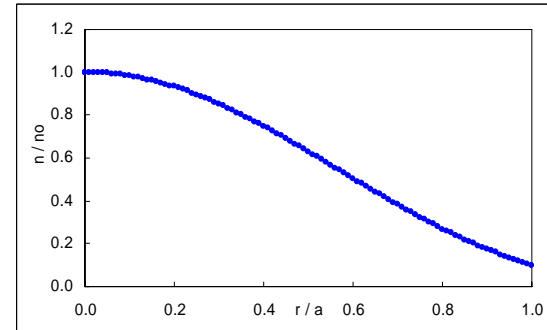

(a)

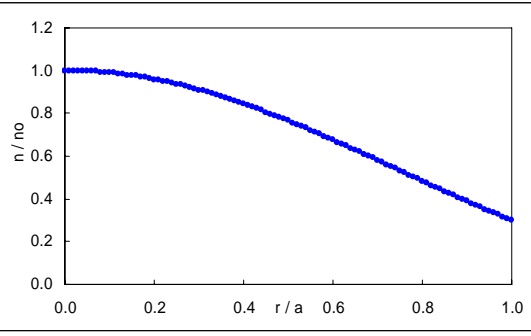

(b)

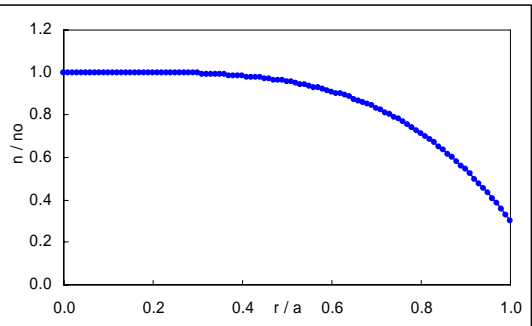

(c)

Fig. 12. Density profiles for $\left[s, t, f_{\mathrm{a}}\right]$ set to (a) $[2,4,0.1]$, (b) $[2,4,0.3]$, and (c) $[4,1,0.3]$. 


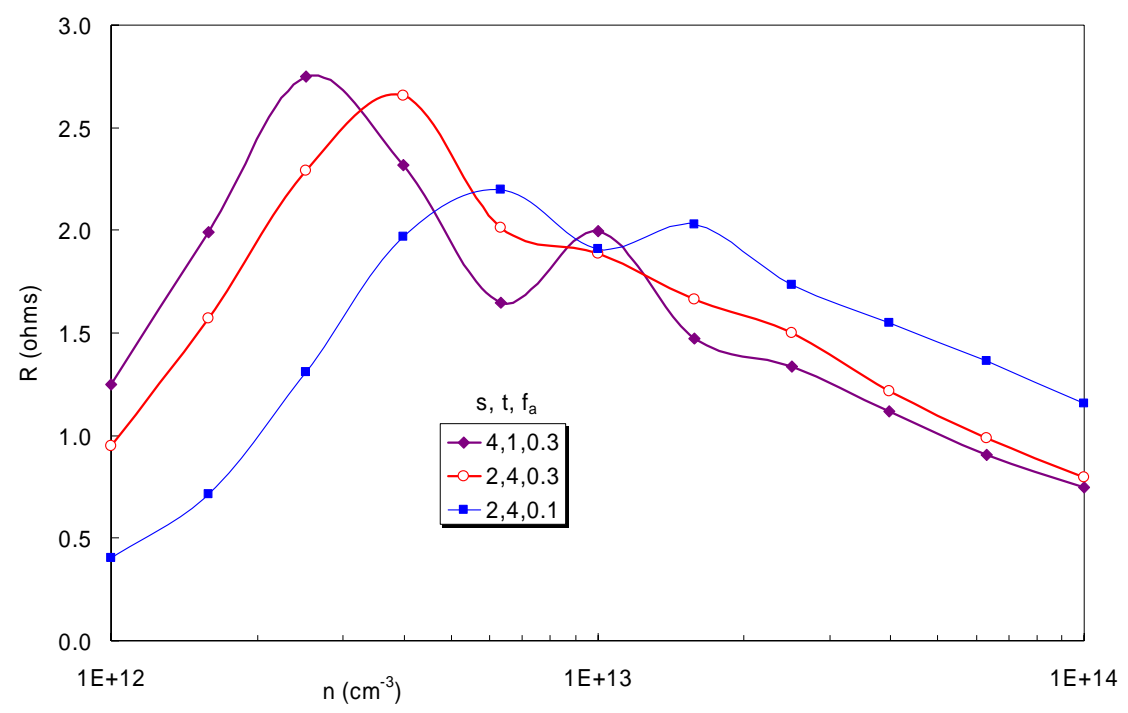

Fig. 13. Variation of $R_{\mathrm{p}}-n$ curves with density profile.

The large edge-absorption also entails a sensitivity to the antenna radius. A small change in the gap $b-a$ will affect the coupling efficiency. In Fig. 14, $R_{\mathrm{p}}$ is plotted for two values of $b$ differing by only $2 \mathrm{~mm}$. A small but significant increase in $R_{\mathrm{p}}$ can be seen when the gap is decreased from 10 to $8 \mathrm{~mm}$. Thus, it is important to make a thin antenna closely wrapped around a thin tube.

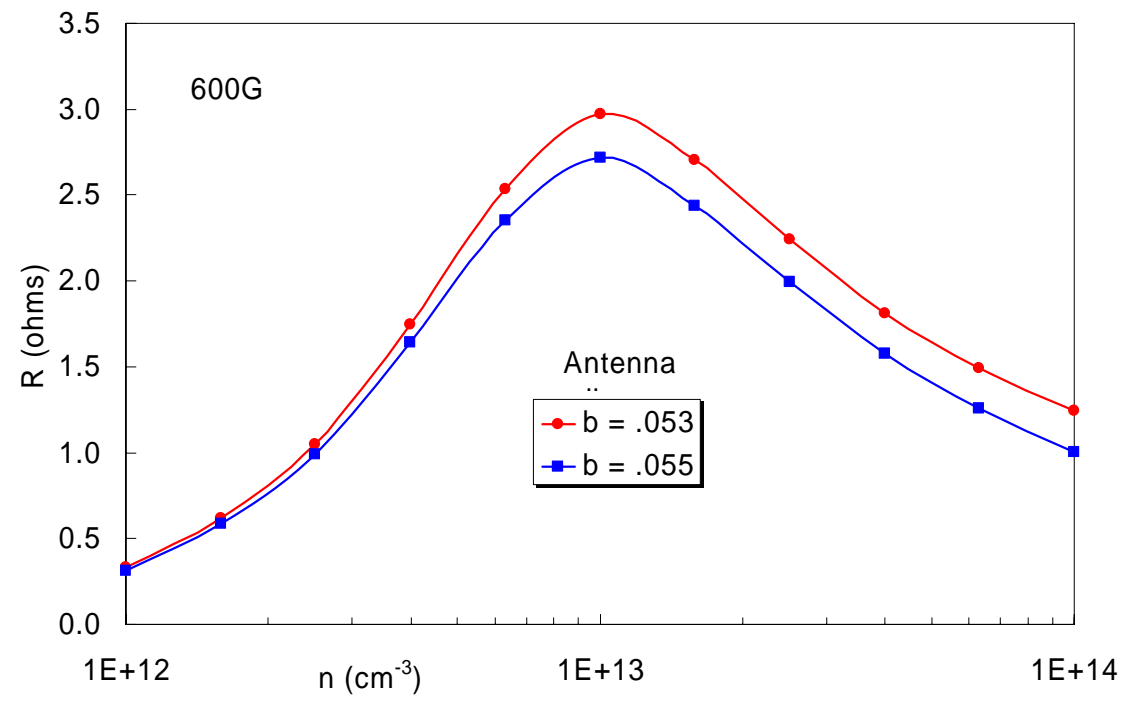

Fig. 14. $R_{\mathrm{p}}$ vs. $n$ for $a=4.5 \mathrm{~cm}, h=5 \mathrm{~cm}$ and two values of the antenna radius.

The use of a conducting endplate permits adjustment of the plasma potential with a DC bias voltage, and it is also easier to add features such as a gas feed line or a cooling tube. If an insulating endplate is used, the wave's E-field does not change sign at that boundary, and our $h$ value of $5 \mathrm{~cm}$ is no longer optimum for the low-field peak, as shown in Fig. 15. However, the peak can usually be recovered by increasing $h$ by about a half-wavelength of the dominant mode. In this particular case, multiple modes are encountered, and the best that can be done is to use a much longer alumina tube. Wall losses to such a tube would greatly decrease the useful density. A conducting endplate is therefore much to be preferred in this design. 


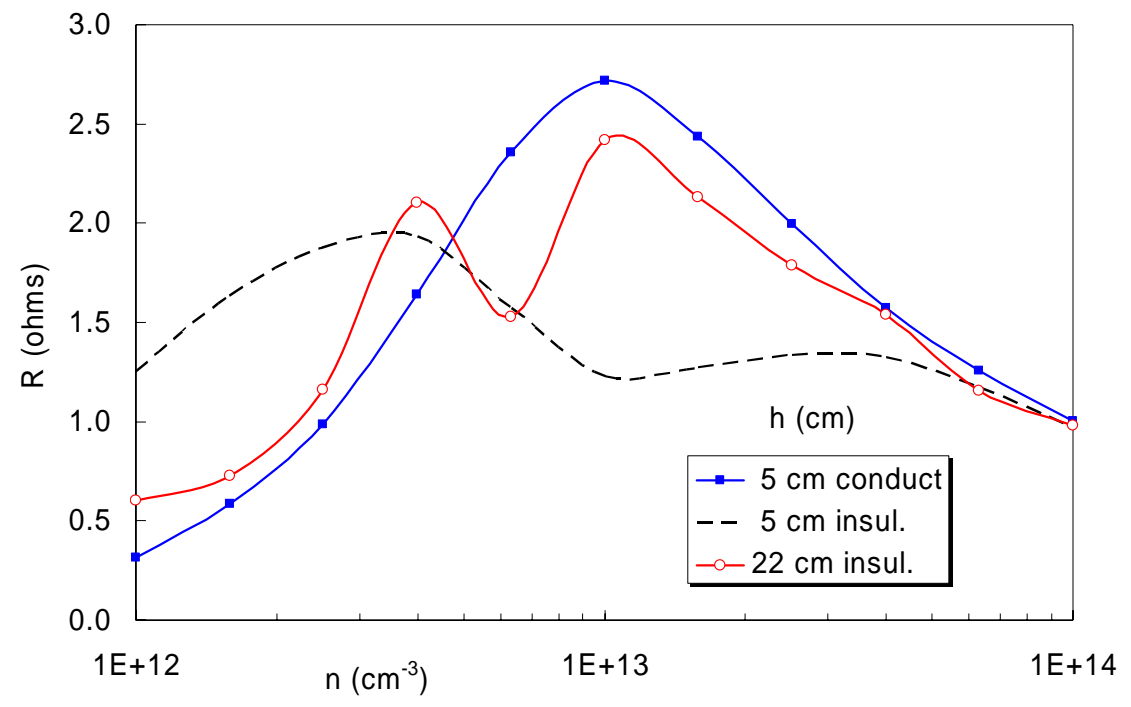

Fig. 15. $R_{\mathrm{p}}$ vs. $n$ for a 9 -cm diam tube, $5 \mathrm{~cm}$ long, at $600 \mathrm{G}$, with a conducting endplate $(\bullet)$ and with an insulating endplate (- - ). The red line $(O)$ is for a $22 \mathrm{~cm}$ long tube with insulating endplate.

We next examine the dependence on RF frequency. Figure 16 shows the loading curves at 400 and $600 \mathrm{G}$ for three frequencies. The data are shown in pairs at each frequency. First, note that, all else being equal, higher $B$ shifts the curves to higher $n$, in agreement with Eq. (1). Second, the curves shift to lower $n$ at higher frequency, also as Eq. (1) predicts. The loading is poor at low frequencies. At $27.12 \mathrm{MHz}$, it would seem that a much higher loading can be achieved, but this occurs at too low a density for our purposes. At $400 \mathrm{G}$, the increase in $R_{\mathrm{p}}$ with $f$ at $n=10^{13} \mathrm{~cm}^{-3}$ is not large and is not needed as long as $R_{\mathrm{p}}$ is well above $1 \mathrm{ohm}$. Furthermore impedance matching may be more difficult, as will be seen in the next section. At $600 \mathrm{G}$, the increase in $R_{\mathrm{p}}$ at $n \geq 10^{13} \mathrm{~cm}^{-3}$ may be worth investigating. This is done in Fig. 17, which shows the $R_{\mathrm{p}}$ curves at $600 \mathrm{G}$ and $27.12 \mathrm{MHz}$ as the tube length is varied. The optimum tube length in this case is a very short $3 \mathrm{~cm}$.

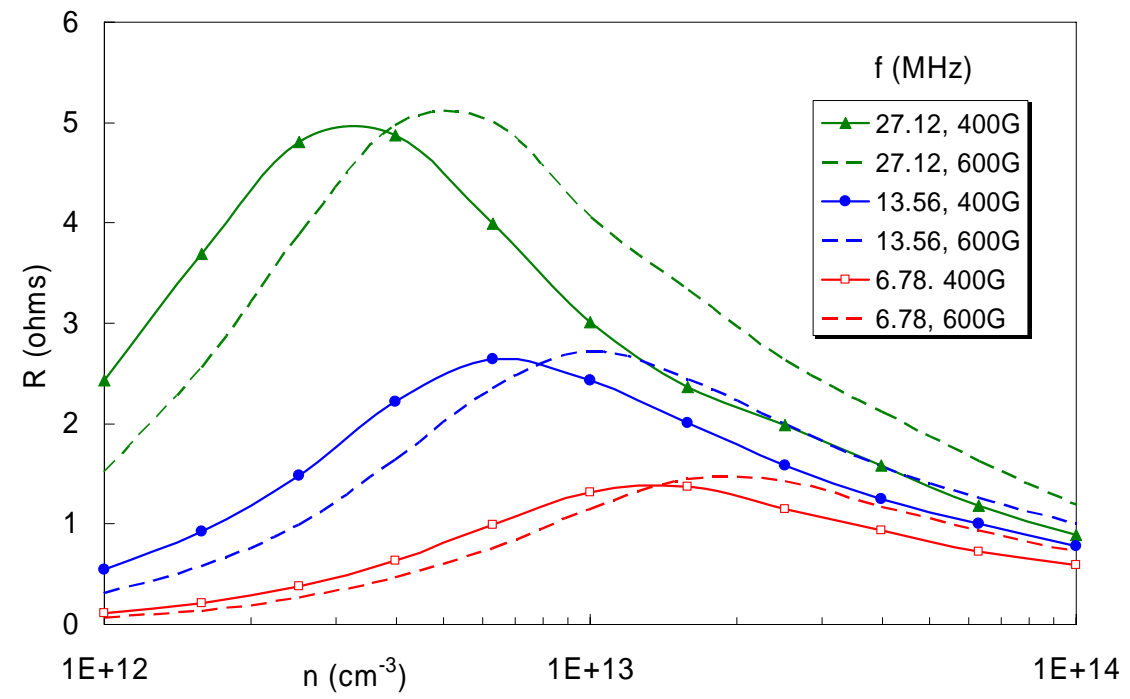

Fig. 16. $R_{\mathrm{p}}-n$ curves at $400 \mathrm{G}$ and $600 \mathrm{G}$ (dashed curves) for a $5 \mathrm{~cm}$ long tube at $6.78 \mathrm{MHz}$ and its harmonics. 


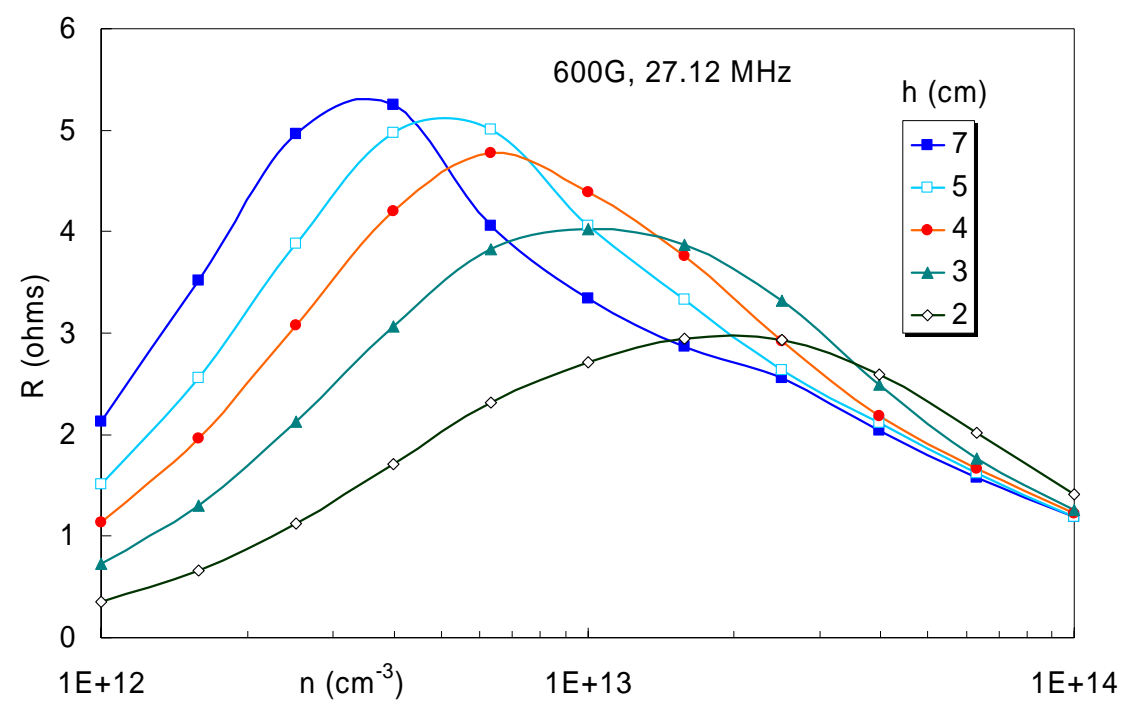

Fig. 17. Variation of $R_{\mathrm{p}}$ with tube length at $27.12 \mathrm{MHz}$ and $600 \mathrm{G}$.

The fact that $R_{\mathrm{p}}$ increases with frequency is due to TG damping. If we keep $n / B$ and the tube dimensions constant in Eq. (1), simple helicon theory ${ }^{42}$ predicts that the damping decrement $\operatorname{Im}(k)$ will not change with $\omega$. TG wave damping ${ }^{43}$, however, increases with its inverse group velocity, which is proportional to $\omega$ when $\omega<<\omega_{\mathrm{p}}, \omega_{\mathrm{c}}$. A study, omitted here, of the wave and deposition profiles for the 13.56 and $27.12 \mathrm{MHz}$ profiles in Fig. 16 indeed shows a large difference in the TG mode behavior.

\section{Design of permanent magnets}

Neodymium Iron Boron (NdFeB) magnets have internal fields exceeding $12 \mathrm{kG}$ and are not only the strongest, but also the most economical in small quantities when tooling cost is included. For plasma processing applications, where B-fields $\leq 100 \mathrm{G}$ are required, the magnets can be very small ${ }^{19}: 7.6 \mathrm{~cm}$ I.D. $\times 12.7 \mathrm{~cm}$ O.D. $\times 2 \mathrm{~cm}$ thick. The field lines of a larger annulus suitable for the 9-cm diam tube are shown in Fig. 18, and the magnitude of $B_{z}$ in the far-field region is given in Fig. 19. Note the stagnation point is close to the magnet. The radial component $B_{\mathrm{r}}$ is negligible throughout, but $B_{\mathrm{z}}$ changes appreciably even in a short tube. The field magnitude and shape in Fig. 19 were calibrated with a gaussmeter. Note that the stagnation point occurs at $z \approx 7 \mathrm{~cm}$, or just $2 \mathrm{~cm}$ from the end of the magnet. The antenna is shown where $B_{\mathrm{z}}$ is $400 \mathrm{G}$. At the endplate the field rises to $600 \mathrm{G}$. This gradient is not deleterious to the discharge but it may affect the curves calculated with HELIC. This error can easily be compensated for by moving the magnet to change the field strength. The field uniformity can be changed by changing the aspect ratio of the magnet. The magnitude of $B_{Z}$ is mainly set by the volume of the magnet. The optimized source, shown in the $400 \mathrm{G}$ position, is shown in Fig. 20.

Although $\mathrm{NdFeB}$ magnets are strong, easy to fabricate, highly resistant to demagnetization, and do not require expensive cobalt, their Curie point is low, leading to a working temperature of only $150 \mathrm{C}$. If temperatures higher than is occur in spacecraft, $\mathrm{SmCo}$ or Alnico magnets can be used. Their remanent B-fields are also above 10kG. SmCo has a 300C working temperature and medium resistance to demagnetization. It is also more resistant to radiation. Alnico will work up to $540 \mathrm{C}$, but it demagnetized more readily than the others. At the experimental stage $\mathrm{NdFeB}$ magnets are clearly the most convenient. 


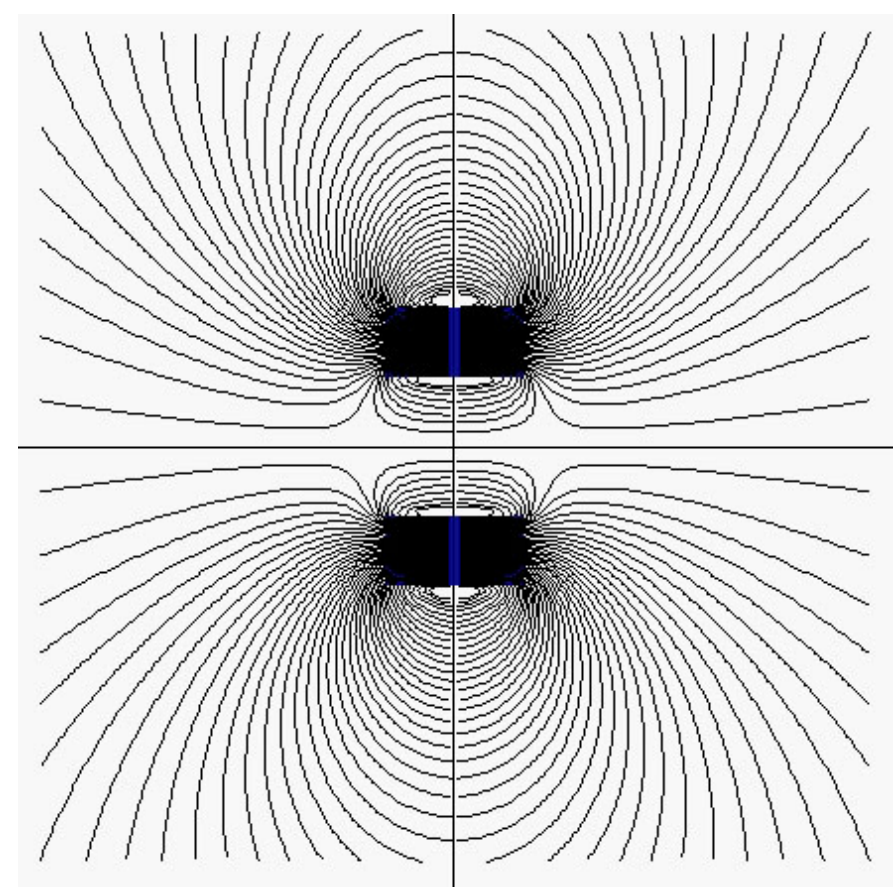

Fig. 18. Field lines in a diametral slice through a ring magnet of $10 \mathrm{~cm} \mathrm{I.D.} \mathrm{by} 20 \mathrm{~cm}$ O.D. and $10 \mathrm{~cm}$ thick. The coordinate $z$ starts at the midplane and increases to the right.

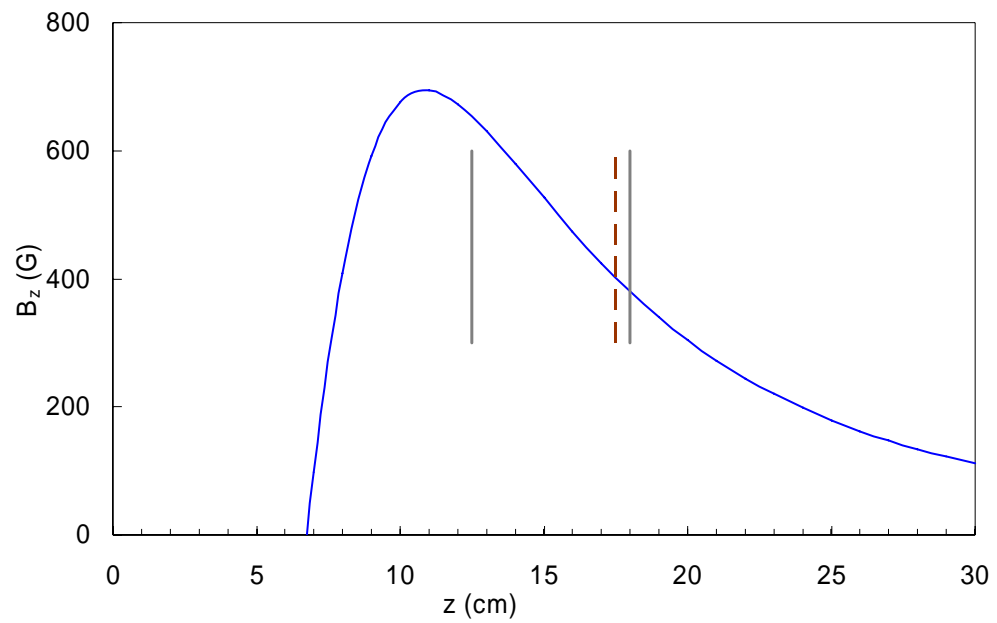

Fig. 19. Magnitude of the $B_{z}$ field on axis in the far-field region of the magnet in Fig. 20. The midplane of the magnet is at the origin. The dashed line is the position of an antenna located at $B_{\mathrm{z}}=400 \mathrm{G}$. The solid lines are the ends of $5-\mathrm{cm}$ long tube with the endplate at the left. 


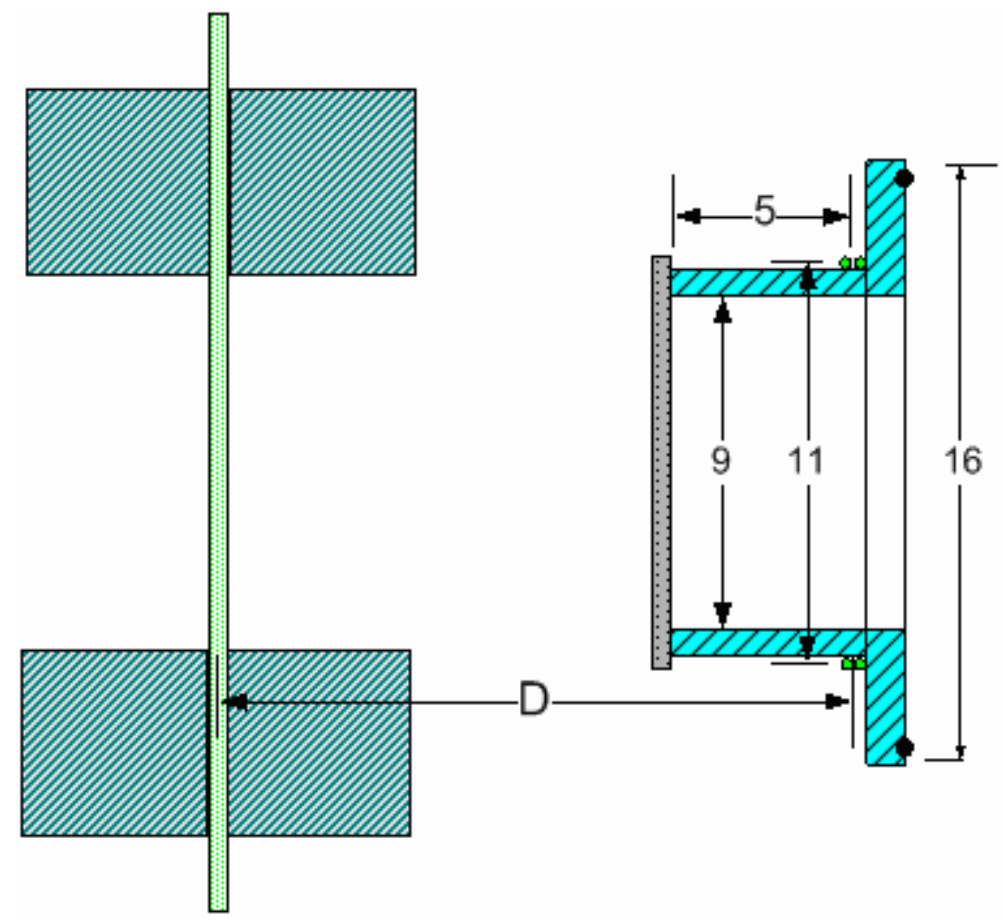

Fig. 20. The optimized 9-cm diam source is shown with dimensions in $\mathrm{cm}$, together with a NdFeB magnet designed for $400 \mathrm{G}$ at the antenna. $D$ is the distance from the midplane of the magnet to the midplane of the antenna. The magnet is made in two pieces supported by a non-ferrous metal plate. The B-field can be adjusted by changing $D$ either by hand or remotely with a motor.

To operate at $600 \mathrm{G}$, the magnet must be much larger. We can increase the length of the magnet, giving rise to Fig. 21a, or increase its diameter, giving Fig. 21b. The longer magnet has the same I.D. and O.D. as in Fig. 20 but is twice as long. The wider magnet has the same length as in Fig. 20 but has a 30-cm O.D. The former is more compact, but the field is more nonuniform and occurs farther from the magnet. The latter has a more gentle $B_{z}$ slope but is very large compared with the discharge. It is shown in Fig. 22.

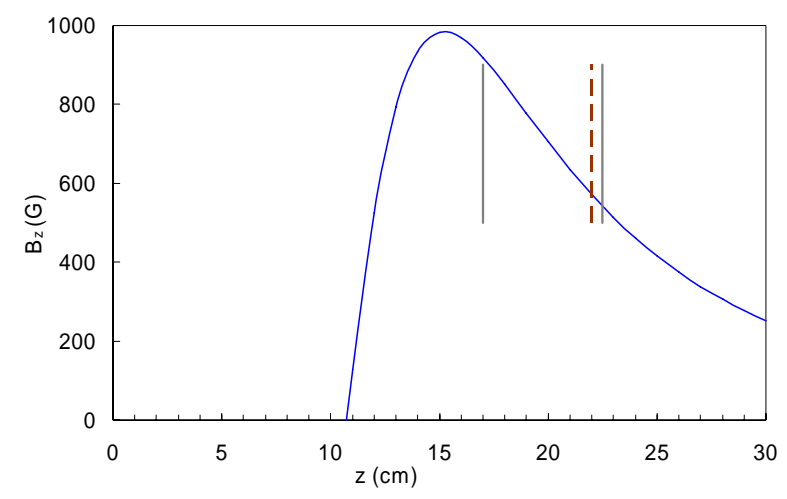

(a)

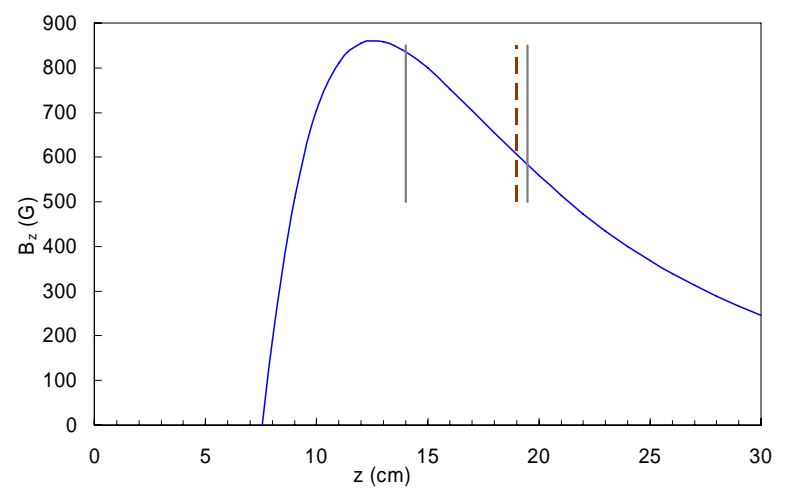

(b)

Fig. 21. $B_{z}$ for (a) a $10 \times 20 \times 20 \mathrm{~cm}$ and (b) a $10 \times 30 \times 10 \mathrm{~cm}$ magnet. The midplane of the magnet is at the origin. 


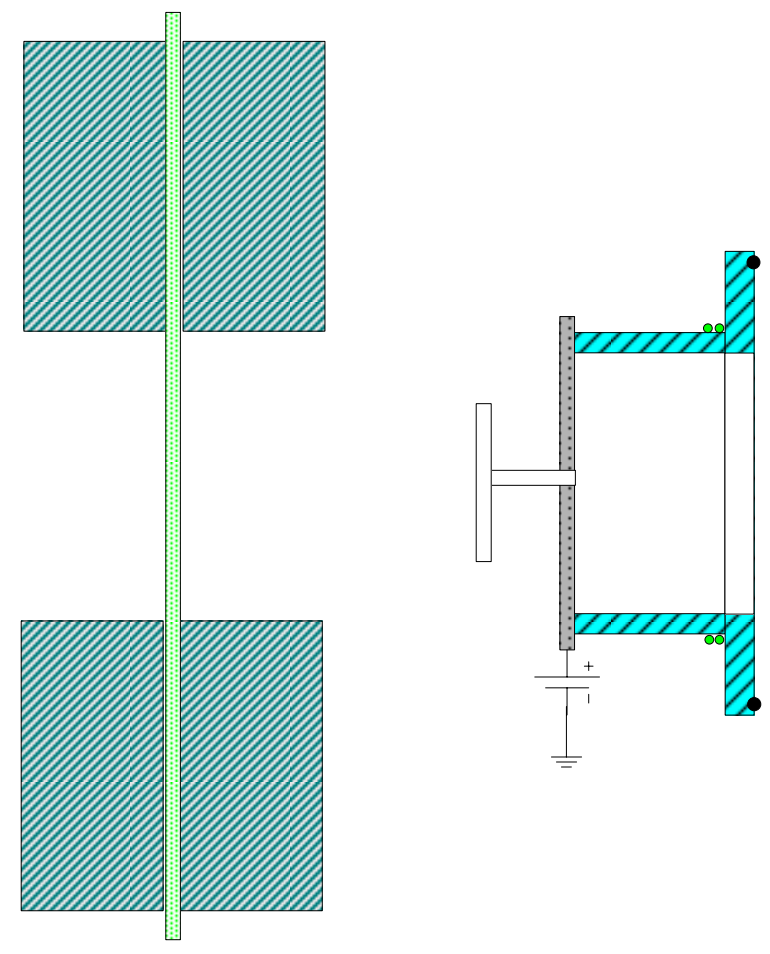

Fig. 22. Layout of magnet and tube for $600 \mathrm{G}$ operation, showing a gas feed line and a DC bias supply.

Such a large magnet would be extremely heavy, and it may not have an advantage over an electromagnet with its DC power supply and cooling system. Either magnet may be impractical and may need shielding. The NeFeB magnet shown in Fig. $5 \mathrm{~b}$ weighs only $0.97 \mathrm{~kg}$, since the HDLT source requires only 60-100G. The 400-G VASIMR magnet in Fig. 20 would weigh $14 \mathrm{~kg}$. A $600-\mathrm{G}$ magnet of the same diameter would weigh $28 \mathrm{~kg}$, while the wider $600-\mathrm{G}$ magnet of Fig. 22 would be $37.7 \mathrm{~kg}$. SmCo magnets of the same sizes would be $38 \%$ heavier. The smaller 600-G experimental device of Sec. VII is much more reasonable.

\section{Engineering considerations}

In RF operation the tube will become quite hot. The ions will strike the wall with an energy of about $5 K T_{\mathrm{e}}$ (the sheath drop), and the electrons with about $2 K T_{\mathrm{e}}$, for a total of $\approx 7 K T_{\mathrm{e}}$. The flux of ion-electron pairs is approximately the Bohm flux $1 / 2 n K T_{\mathrm{e}}$. A conservative estimate can be made by inserting this energy flux into the Stefan-Boltzmann law, neglecting convective cooling and radiation from the inside surface. For $K T_{\mathrm{e}}=3 \mathrm{eV}$ and $n=2 \times 10^{13} \mathrm{~cm}^{-3}$, the wall temperature is $\approx 860^{\circ} \mathrm{C}$, well within the limits of quartz and alumina, but not pyrex. Although the assumption of black-body radiation is optimistic, radiation varies as $T^{4}$, so that low emissivity will incur only a small rise in wall temperature. The antenna is easily made from $3.18 \mathrm{~mm}$ O.D. copper tubing wound tightly around the discharge tube. The water flow through the tubing is more than sufficient to cool the antenna.

The following sections contain details of the design of the matching circuit, details which may appear to be trivial or irrelevant; but this is not the case. There is a sweet spot for this type of source where the tube diameter, antenna design, transmission line, and rf frequency can be adjusted so that they all work together. For instance, if the tube is too wide, the antenna inductance is too large to be matched at $13.56 \mathrm{MHz}$, and the current needed to power the larger plasma would lead to higher circuit losses. 
A standard two-capacitor matching circuit can be designed with the analytic formulas given by $\mathrm{Chen}^{28}$. Here $\mathrm{C} 1$ is the capacitor nearer the power supply and is the loading capacitor in the Standard $(\mathrm{S})$ configuration. $\mathrm{C} 2$ is the capacitor nearer the antenna and is the tuning capacitor in the $\mathrm{S}$ configuration. The Alternate (A) configuration has $\mathrm{C} 1$ as the tuning capacitor and $\mathrm{C} 2$ as the loading capacitor; it is not shown here because it is less convenient in this case. The inductance L of the antenna is small and difficult to measure, but it can be "backed out" from the analytic formula by measuring $\mathrm{C} 1$ and $\mathrm{C} 1$ when matched. For a 3-turn antenna on a 5cm I.D. tube, $\mathrm{L}$ is about $0.7 \mu \mathrm{H}$, which translates to $1.24 \mu \mathrm{H}$ on a 9-cm diam tube. Unless otherwise specified, the curves in Fig. 23 are computed for $\mathrm{L}=1.24 \mu \mathrm{H}, R=2 \Omega, Z=60 \mathrm{~cm}, f=$ 13.56 MHz, and 50- $\Omega$ air-core rigid coax. Here $R$ is the combined antenna, cable, and plasma resistance; and $Z$ is the cable length between the matching circuit and the antenna.

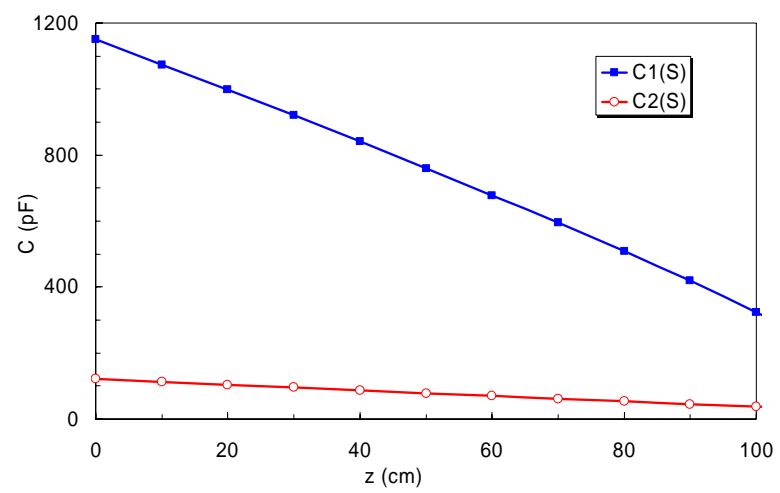

(a)

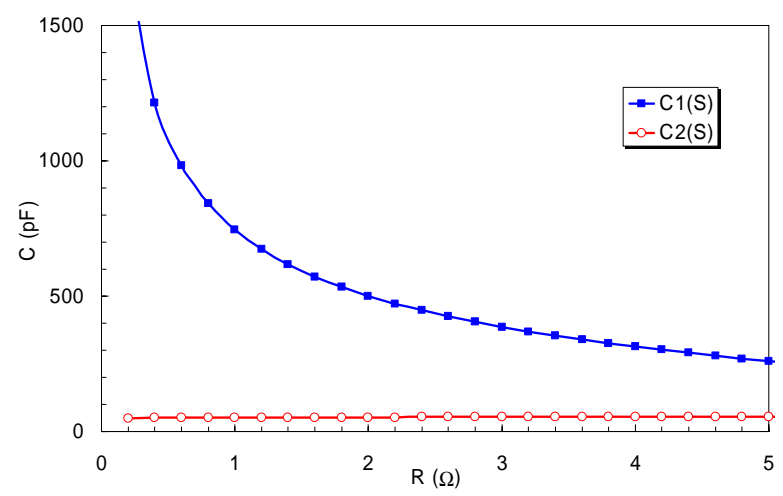

(b)

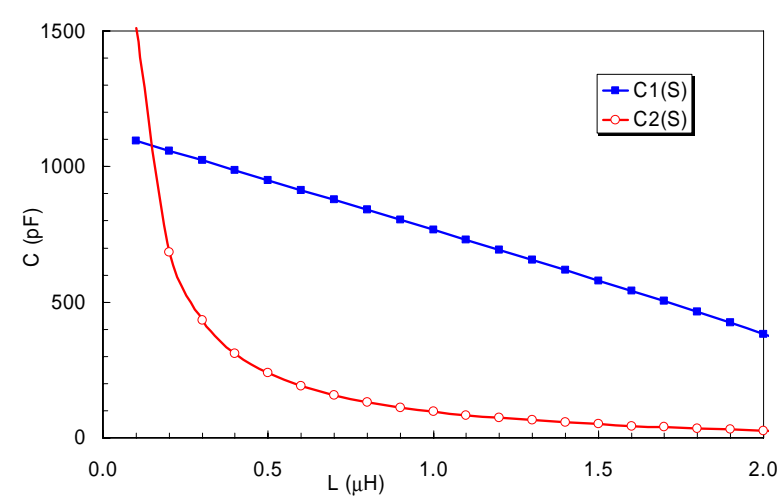

(c)

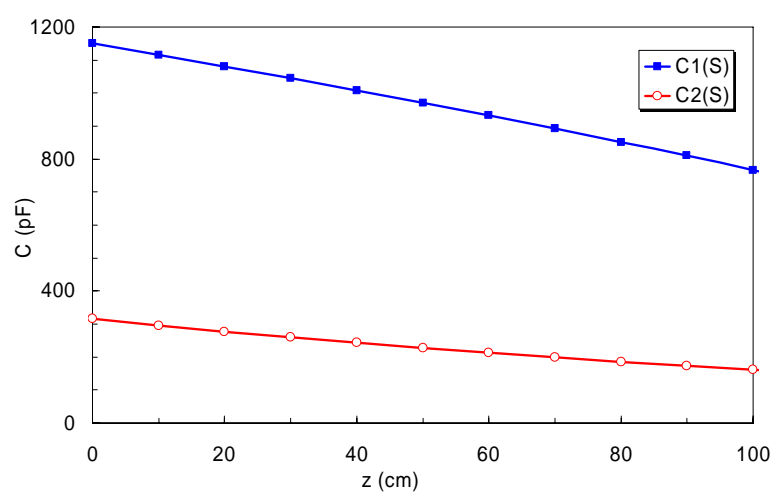

(d)

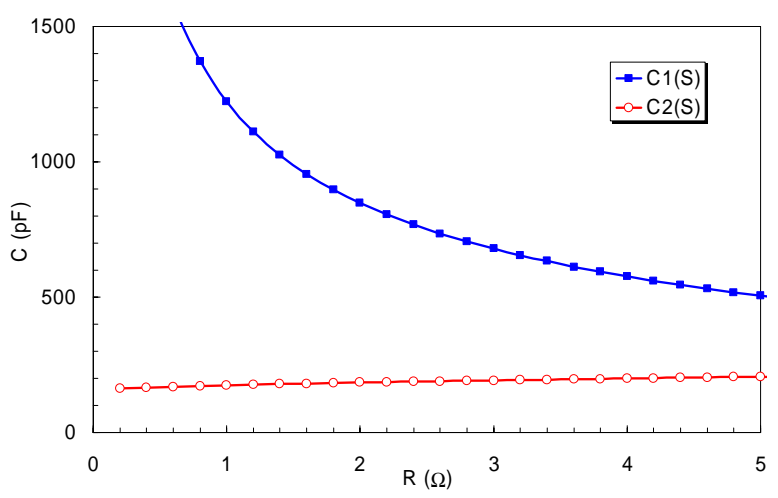

(e)

Fig. 23. Matching circuit capacitances for a 3-turn $m=0$ antenna (a-c) and a 2-turn antenna (c-e) on a 9-cm I.D. tube. In (c) the antenna $\mathrm{L}$ is varied for constant $R_{\mathrm{p}}$ and $\mathrm{Z}$.

The cable length $Z$ is critical if it has to be long. In multi-tube sources long cable lengths are necessary if all tubes are powered from the same matching circuit. Here the solution is not 
difficult since only one tube is involved, and the cable-length restrictions can usually be met. In Fig. 23a, there is no solution if $Z>130 \mathrm{~cm}$; the 2-turn antenna in Fig. $23 \mathrm{~d}$ is less restrictive. If long connection lengths are necessary, there is an upper limit on the inductance L. In Figs. 23b and e, we see that $\mathrm{C} 1$ becomes less sensitive if $R$ is larger than about $2 \Omega$, which we tried to achieve in the tube design. The range of $\mathrm{C} 2$ is more convenient in Fig. 23e for the 2-turn antenna. Figure $23 \mathrm{c}$ shows that $\mathrm{C} 2$ becomes inconveniently small if $\mathrm{L}$ is much larger than $1 \mu \mathrm{H}$.

The matching conditions at $27.12 \mathrm{MHz}$ are shown in Fig. 24. As expected, all capacitances are lower. A two-turn antenna with $\mathrm{L} \approx 0.55 \mu \mathrm{H}$ is assumed. A transmission line with $Z=60 \mathrm{~cm}$ is close to the limit of $70 \mathrm{~cm}$. There is no solution for $R>3.5 \Omega$ or $\mathrm{L}>0.6 \mu \mathrm{H}$. These restrictions are relieved if $Z=30 \mathrm{~cm}$, but this may be too short to be practical. A one-turn antenna with small L could be matched more easily, but a very large current would have to be carried by the transmission line. These matching difficulties may be circumvented by using ferrite transformer coupling, as proposed by Rayner et al. $^{44}$, and by Godyak ${ }^{45}$. However, only one standard ferrite compound can handle frequencies up to $30 \mathrm{MHz}$.

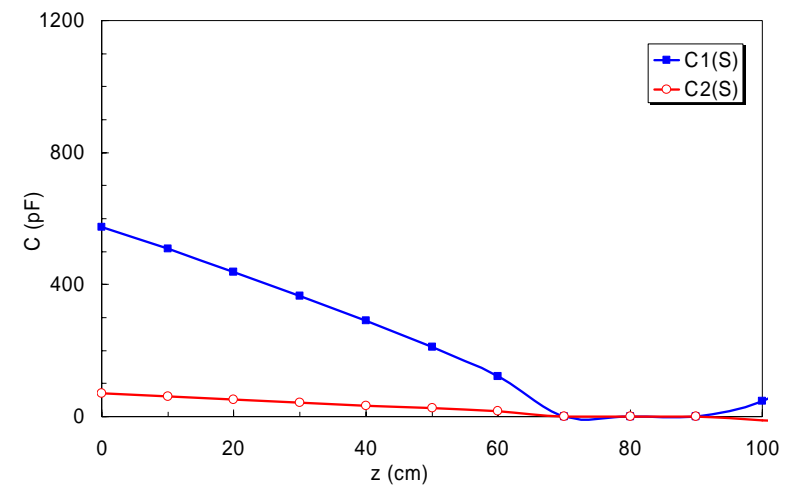

(a)

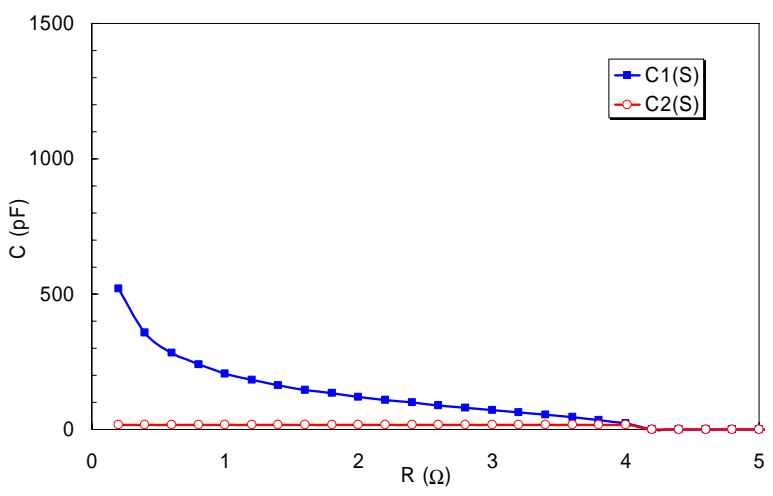

(b)

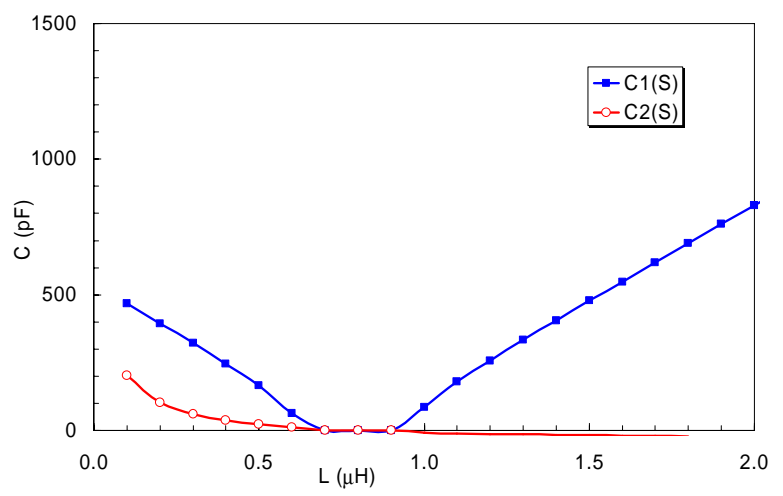

(c)

Fig. 24. Same as Fig. 23, but for $f=27.12$ MHz.

The cabling between the matching network and the antenna has to handle the voltage under no-load or low-density conditions and the current in RF operation. Suppose $\mathrm{L}=1.24 \mu \mathrm{H}$, $R_{\mathrm{c}}=0.25 \Omega$, and $f=13.56 \mathrm{MHz}$. The antenna's inductive impedance is $106 \Omega$, and $I$ and $V$ will be nearly $90^{\circ}$ out of phase. Before breakdown, if the power supply is set for $5 \mathrm{~kW}$, the peak voltage will be $1028 \mathrm{~V}$, and the rms current $141 \mathrm{~A}$. After breakdown, if $R_{\mathrm{p}}=3 \Omega$, the rms current will be 41A. Normal coaxial cables such as RG393 with $\mathrm{N}$ or HN connectors will not be able to handle these loads without overheating. Thus, cooled rigid coax has to be used, and the joints have to be soldered. The inner conductor can be a tube carrying water to the antenna. To avoid large RF currents in the water line, it can be coiled into an inductor. 
We consider next the power necessary to produce a given density. Argon is more easily ionized than hydrogen or helium, and the power computed here for argon will be an underestimate. Xenon, however, will require less power than argon. The loss rate is controlled by the ions, which are not confined and escape to the walls and ends, of total area ${ }^{46} 2 \pi a(a+L)$. If the density profile is known, the ion flux can be calculated by diffusion theory at high pressures, or by the Bohm flux at the sheath edge at low pressures. Since $n(r)$ has not been measured for this configuration, we had to assume a density profile (Fig. 12b). The ion flux is then the Bohm flux at the acoustic velocity of $1.7 \times 10^{5} \mathrm{~cm} / \mathrm{sec}$ for argon at $K T_{\mathrm{e}}=3 \mathrm{eV}$ at the assumed edge density. Each electron-ion pair that is lost requires an energy

$$
W=W_{i}+W_{e}+E_{C}
$$

to replenish, where $W_{\mathrm{i}}$ and $W_{\mathrm{e}}$ are the ion and electron kinetic energies, and $E_{\mathrm{c}}$ is the ionization and radiation energy lost in inelastic collisions. $W_{\mathrm{i}}$ is of order $5.5 K T_{\mathrm{e}}$, of which $0.5 K T_{\mathrm{e}}$ is the Bohm velocity at the sheath edge, and $5 K T_{\mathrm{e}}$ is the sheath drop. $W_{\mathrm{e}}$ is of order $2 K T_{\mathrm{e}}$, which includes both the parallel and transverse energies of the electron striking the wall. $E_{\mathrm{c}}$, which contains the ionization and inelastic cross section information, has been computed by several authors and is given by Lieberman and Lichtenberg ${ }^{47}$ for argon and oxygen, but not for light atoms. For argon, this computation yields an energy loss rate of

$$
P_{\text {out }}=P_{\text {in }} \approx 3.8 \times 10^{-10} n=3800 \text { Watts }
$$

if $n=10^{13} \mathrm{~cm}^{-3}$. Here $n$ is the density near the wall but before the sheath edge. Since we chose a profile with an edge fraction [Eq. (2)] of 0.3, the peak density is $3.3 \times 10^{13} \mathrm{~cm}^{-3}$. This is of course a very rough estimate, since the actual density profile cannot be found without knowing the depleted neutral density and its profile. This type of calculation, however, has yielded a power-density relationship in agreement with measurements within experimental error ${ }^{39}$. For hydrogen or helium, RF energy much higher than $3.8 \mathrm{~kW}$ will be needed to achieve this density.

\section{Design of a 5-cm diameter high-power source}

For experiments on these high-power sources, it may be convenient to construct a smaller device of 5-cm I.D. The $R_{\mathrm{p}}-n$ curves for this diameter and various antenna distances $h$ are shown in Fig. 25a for $B=600 \mathrm{G}$. Since a thinner tube can support atmospheric pressure, the gap $b-a$ has been reduced from 10 to $8 \mathrm{~mm}$. The values of $R_{\mathrm{p}}$ are lower than for the $9-\mathrm{cm}$ diam case because the volume of absorbing plasma is smaller. Variation of $B$ simply shifts the curves horizonatally according to Eq. (1) without changing the magnitude. However, the larger value of $k_{\mathrm{r}}$ in Eq. (1) permits using a larger $\omega$, and this increases the absorption, as is seen in Fig. 25b. Note that the $R_{\mathrm{p}}$ scale is twice as large as in Fig. 25a. The best value of $h$ seems to be $3 \mathrm{~cm}$, for which $R_{\mathrm{p}}$ is above $2 \Omega$ over a wide range of $n$ near and above $10^{13} \mathrm{~cm}^{-3}$.

The wave and deposition characteristics of this optimized discharge are shown in Fig. 26. Figure 26a shows that there is one dominant axial mode. The radial profile of $\left|B_{z}\right|$ is shown in Fig. 26b together with the radial deposition profile $P(z)$. Again we see that the deposition is dominated by the TG mode in spite of its smaller amplitude. The axial profiles are shown in Fig. 26c. The wave amplitude shows ripples due to interference between the direct and reflected waves. An appreciable amount of power deposition (r.h. scale) occurs downstream of the antenna. 


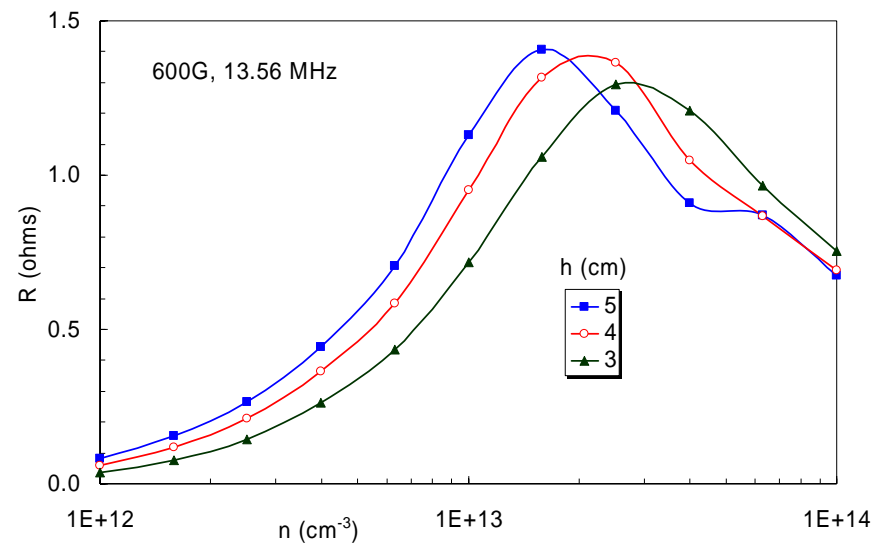

Fig. 25a. Loading curves for a 5 -cm diam tube of various lengths at $13.56 \mathrm{MHz}$ and $600 \mathrm{G}$.

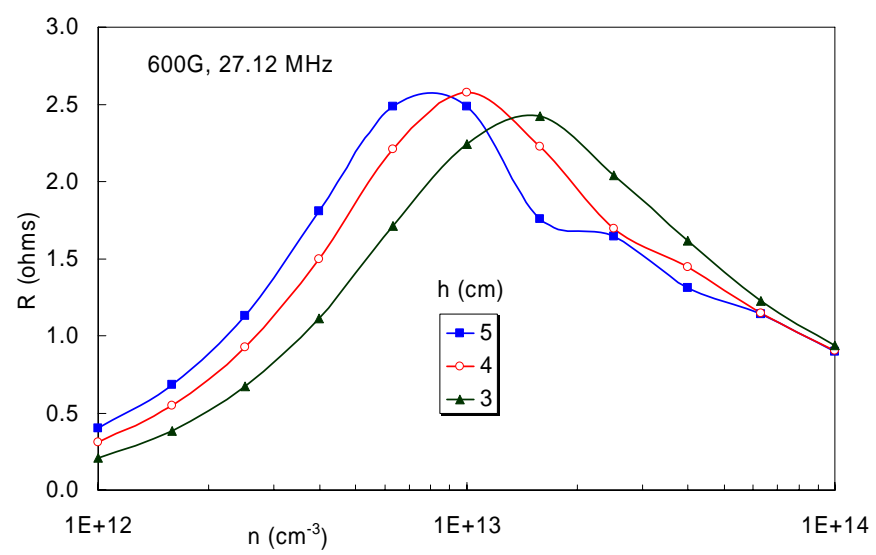

Fig. 25b. Same as (a) but for $27.12 \mathrm{MHz}$.

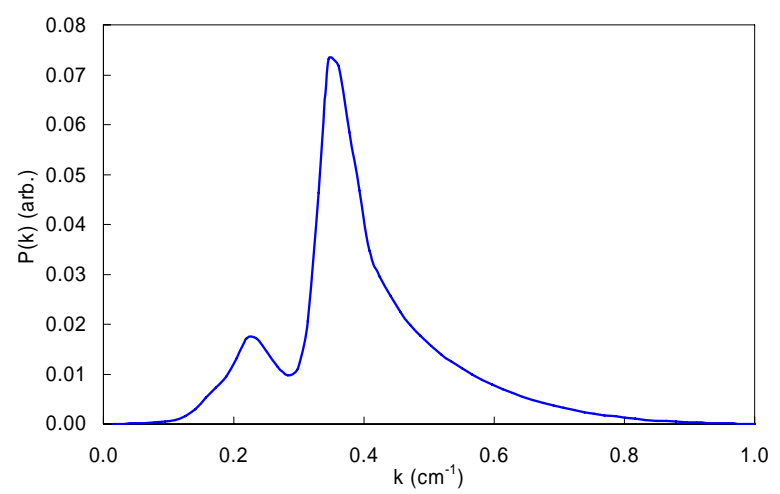

(a)

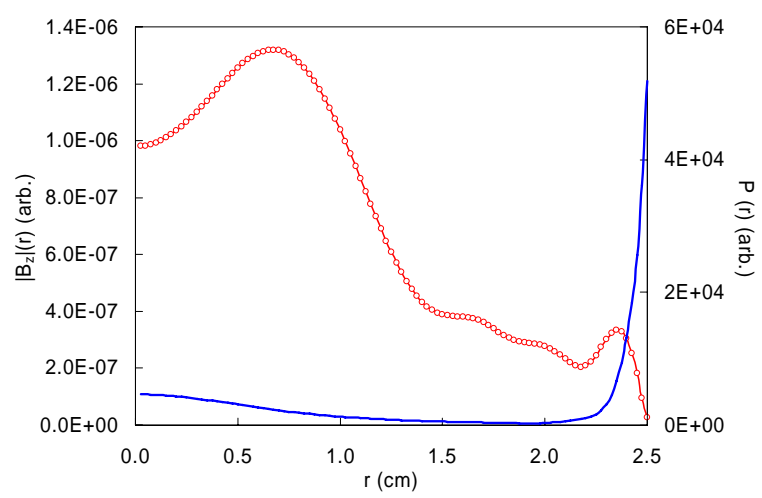

(b) 


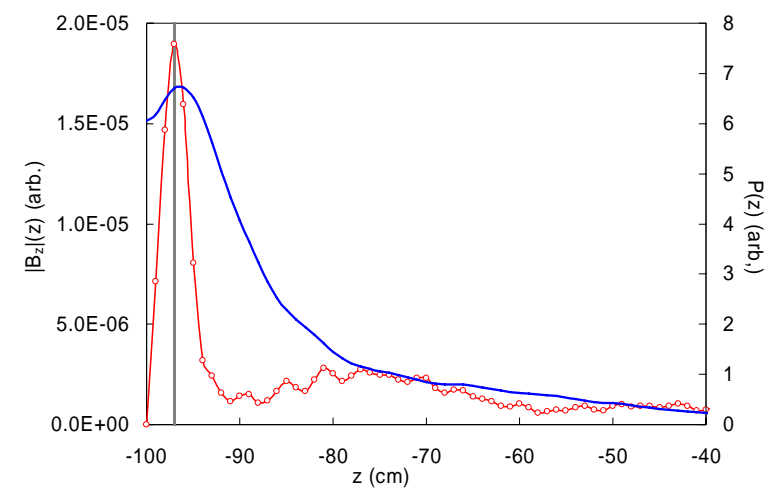

(c)
Fig. 26. (a) $k$-spectrum and (b,c) radial and axial profiles of wave amplitude $(O)$ and deposition rate ( - ) for a 5-cm diam, 3-cm long tube at 27.12 Mhz and 600G. The vertical line in (c) is the antenna position.

To produce a $600-\mathrm{G}$ external field, a large magnet volume is still required, but the smaller diameter is helpful. A possible magnet can contain four rings $7 \mathrm{~cm}$ in I.D., $13 \mathrm{~cm}$ in O.D. and 4 $\mathrm{cm}$ thick, weighing $2.3 \mathrm{~kg}$. Its external field is shown in Fig. 27. The antenna is about $15 \mathrm{~cm}$ from the midplane of the magnet, and the field at the endplate rises to $900 \mathrm{G}$. A diagram of the system is shown in Fig. 28.

To operate at $27.12 \mathrm{MHz}$, the 5 -cm diam system must have a 2-turn antenna with an approximate inductance $\mathrm{L}$ of $0.31 \mu \mathrm{H}$, as will be seen below. The tuning curves are shown in Fig. 29, plotted on the same scale as Figs. 23 and 24 . A cable length of $60 \mathrm{~cm}$ and a load resistance of $3 \Omega$ is assumed unless it is varied. Figure $29 \mathrm{a}$ shows that the cable length is limited to about $1 \mathrm{~m}$. Figure $29 \mathrm{~b}$ shows that $\mathrm{C} 1, \mathrm{C} 2$ are not sensitive to $R$ if $R$ is larger than about 2-3 $\Omega$. Figure $29 \mathrm{c}$ shows that a 3-turn antenna with $\mathrm{L}=0.7 \mu \mathrm{H}$ cannot be matched without other elements.

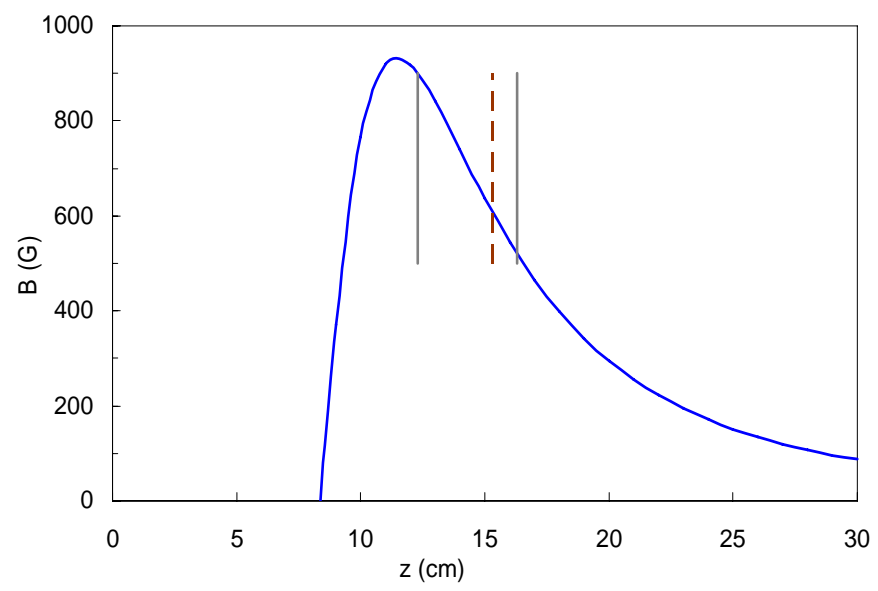

Fig. 27. External B-field of a NeFeB magnet for a 5-cm diam discharge. The dashed line is the antenna position at $600 \mathrm{G}$, and the solid lines are the ends of a $4 \mathrm{~cm}$ long tube. 


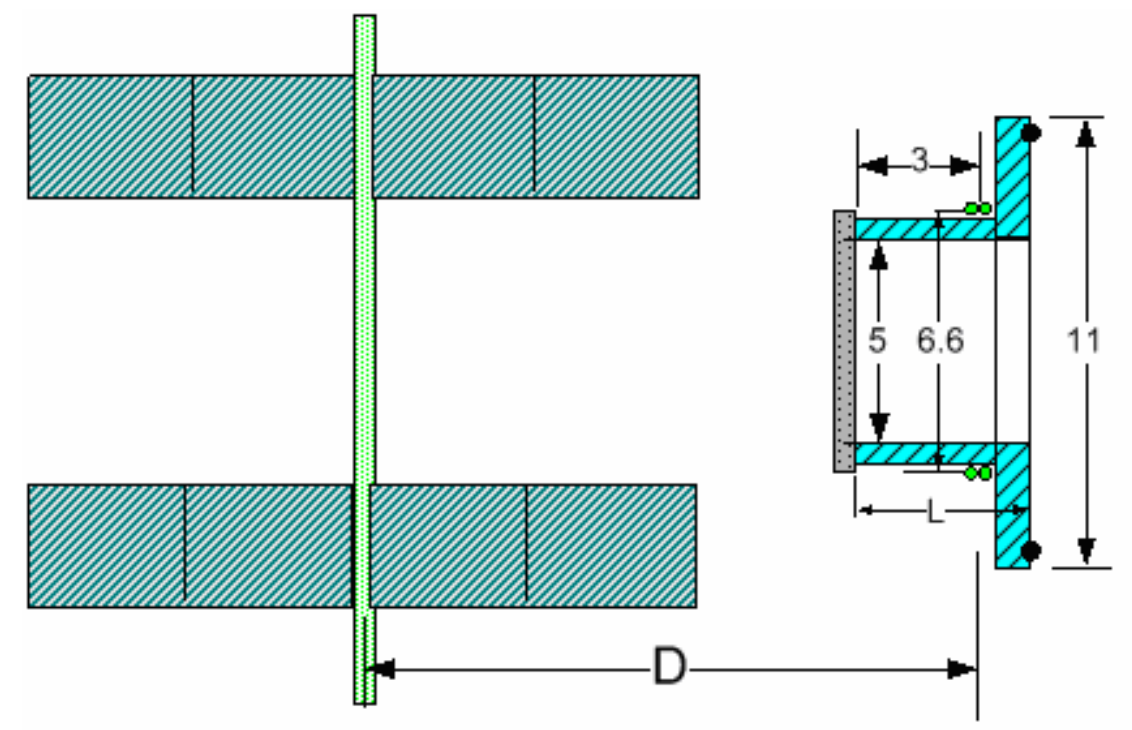

Fig. 28. A 5-cm diam helicon tube and a 600-G magnet designed for a small overall system diameter.

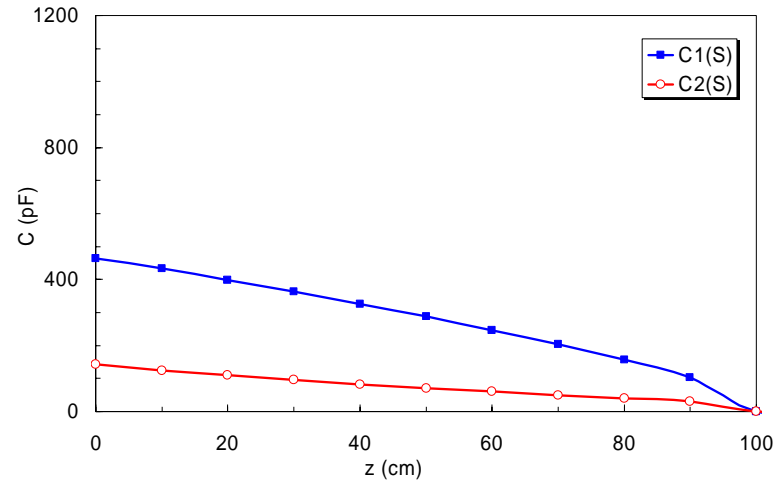

(a)

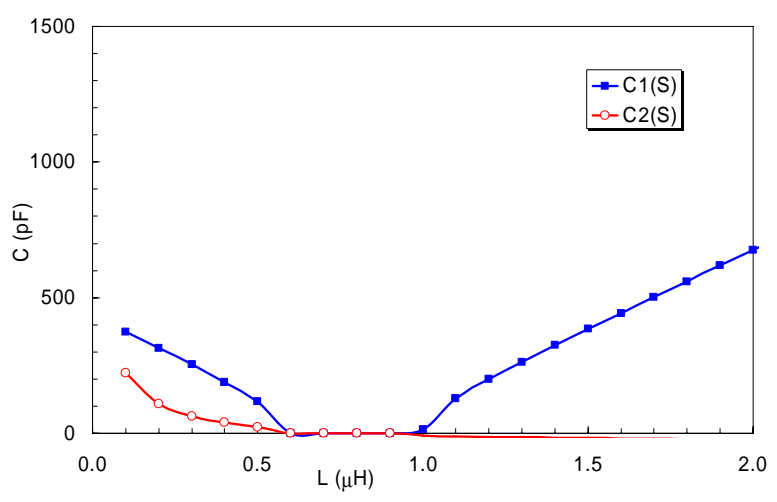

(c)

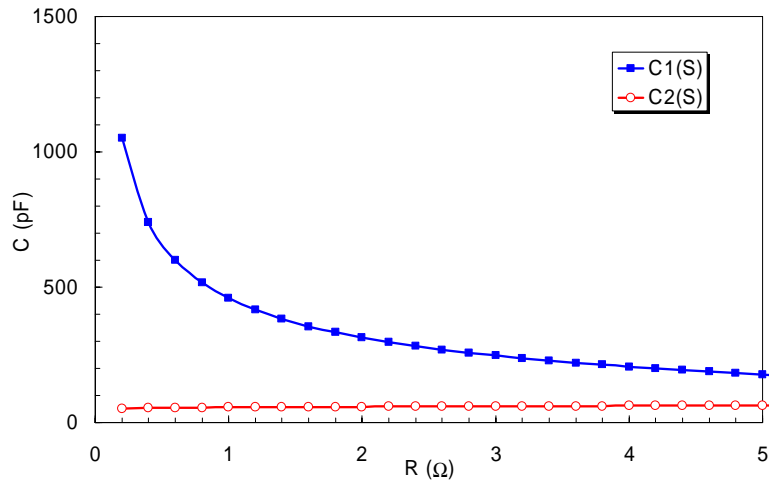

(b)

Fig. 29. Matching network capacitors $\mathrm{C} 1$ and $\mathrm{C} 2$ for a $5-\mathrm{cm}$ diam discharge operating with a 2-turn $m=0$ antenna at $27.12 \mathrm{MHz}$. 


\section{Summary and discussion}

A high-density, permanent magnet helicon plasma source originally designed for plasma processing was modified for operation in an ion thruster. Accuracy of the predictive capabilities of the HELIC code had been shown by experiment. For the Helicon Double Layer Thruster, a very compact source already tested can be adopted directly. For the VASIMR, two systems, of 9-cm and 5-cm plasma diameter, have been designed to produce densities $\geq 10^{13} \mathrm{~cm}^{-3}$ in the source. The aspect ratio of the plasma tube and of the magnet, as well as the antenna and matching network, were optimized. To tune the source for highest density, the B-field can easily be adjusted by moving the magnet. Compared with existing helicon sources, PM helicon sources are lighter and more compact. The helicon source is a complicated device, and the physical mechanisms that make it work have taken over 15 years to decipher. Although we have tried to explain the physics at each step, the methodology may be hard to understand, since the experimental difficulties, especially in $\mathrm{CW}$ operation, were encountered and solved only after several years of operation. The HELIC code was devised in concert with experiment at all stages.

Whether or not this type of source can be used as a thruster can be answered only after extensive testing such as has been done for standard thruster using grid acceleration. This paper is intended to give a head start to such testing with PM helicon sources. The most important questions seem to be: 1) How is the plasma detached from the field lines? 2) Is neutralization with another electron source necessary? 3) How does one compute the trust from a non-uniform ion beam? and 4) What is the gas efficiency?

1) Although electrons have small Larmor radii and one would expect them to be entrained by the B-field, they cross the field lines quite freely in discharges with endplates because of the Simon short-circuit effect ${ }^{48}$. This effect is not well known except to aficionados of magnetized discharges and comes about as follows. The sheath at the endplate has an average potential drop such that electrons and ions leave the plasma at the same rate. On a conducting endplate, however, the sheath drop can vary from point to point while keeping the proper average value. For instance, if the sheath is slightly thinner on field line A, more electrons will be lost there than the average. If it is slightly thicker on a neighboring field line $\mathrm{B}$, fewer electrons will be lost there, and the electron density will be higher. Electrons will have apparently moved from line A to line $\mathrm{B}$, though they never crossed field lines. This mechanism preserves the Boltzmann relation so that the plasma potential $V_{\mathrm{s}}$ can follow $\ln n_{\mathrm{e}}$, as is almost always observed. If the radial E-field were ambipolar, it would point inward, so as to push electrons out and keep ions in; but in helicon discharges it points outward, as the Boltzmann relation requires if the density peaks on axis. As a result, ions diffuse at their normal rate, and electrons are able to follow them to preserve quasineutrality.

The ions diffuse mainly by charge exchange collisions with the neutrals. When they exit the discharge tube, they already have a drift of the order of the sound speed $c_{\mathrm{s}}$ in the $Z$ (horizontal) direction because the Bohm sheath criterion requires it if the potential is to fall monotonically (as long as downstream ionization is much less than that inside the tube). Between the source and the double-layer, ions are further accelerated by the conversion of perpendicular to parallel energy in the diverging B-field ${ }^{13}$. Observations by Charles et al. ${ }^{12}$ show that the ion beam reaches an energy of the order of $50 \mathrm{eV}$ relative to the source. The field of a magnet such as that in Fig. 28 falls to $10 \mathrm{G}$ at $60 \mathrm{~cm}$ from the magnet. At that field, 50-eV argon ions have a Larmor radius of $40 \mathrm{~cm}$, and so are effectively detached from the B-field. Thermal electrons trapped between the endplate and the DL collide with the endplate at about $1 \mathrm{MHz}$ and therefore can follow the ions as they stream out and diverge. Any charge imbalance in the radial 
direction will lead to an azimuthal electric field, and the worst that can happen is that the ion beam gains an azimuthal velocity. This energy comes from the electron distribution and does not affect the directed ion energy.

2) Even after the density falls to $10^{6} \mathrm{~cm}^{-3}$, the Debye length is still small, of the order of $1 \mathrm{~cm}$; and electrons still have to maintain quasineutrality. Thus the electrons follow the ion beam, and no separate electron injector is necessary.

3) In gridded thrusters, uniformity of the ion beam is required to preserve the grids. Here, there is no problem with a peaked ion distribution. The thrust is the integral of the ions' $z$ momenta over the ion beam. Fruchtman ${ }^{49}$ has shown theoretically that the ions can gain no momentum at the double layer. This may be true, but the spacecraft nevertheless gains that momentum at other places. The thrust is ultimately applied at the endplate of the discharge. This is the reason that the ion beam profile is irrelevant.

4) This is best determined by experiment. At the high RF powers considered here, the plasma is almost fully ionized, but the "almost" has to be measured.

The PM helicon source has been patented ${ }^{20}$, but the project was started by a grant from the National Science Foundation. Consequently, non-commercial, government-sponsored research on these ideas should be permissible.

\section{Photograph and biosketch}

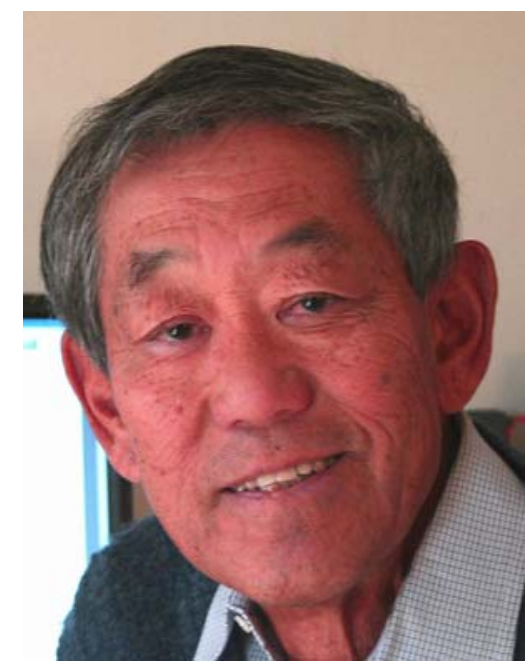

\section{Francis F. Chen (SM'72-F'80-LF'03).}

In his 54-year career in plasma physics, he has devoted about a decade each to the subfields of magnetic fusion, laser fusion, basic plasma physics, plasma diagnostics, and low-temperature plasma physics. $\mathrm{He}$ is the author of Introduction to Plasma Physics and Controlled Fusion and co-author of Principles of Plasma Processing. He chaired the APS Division of Plasma Physics in 1983 and won the IEEE Plasma Science and Applications Award in 1994 and the APS Maxwell Prize in 1995. Formally retired in 1994, he no longer teaches but still works full time on research and writing. 


\section{REFERENCES}

${ }^{1}$ S.A. Cohen, N.S. Siefert, S. Stange, R.F. Boivin, E.E. Scime, and F.M. Levinton, Ion acceleration in plasmas emerging from a helicon-heated magnetic-mirror device, Phys. Plasmas 10, 2593-2598 (2003).

${ }^{2}$ C. Charles and R. Boswell, Current-free double-layer formation in a high-density helicon discharge, Appl. Phys. Lett. 82, 1356-1358 (2003).

3 C. Charles, Hydrogen ion beam generated by a current-free double layer in a helicon plasma, Appl. Phys. Lett. 84, 332-334 (2004).

${ }^{4}$ C. Charles and R.W. Boswell, Laboratory evidence of a supersonic ion beam generated by a current-free “helicon” double-layer, Phys. Plasmas 11, 1706-1714 (2004).

${ }^{5} \mathrm{C}$. Charles, Spatially resolved energy analyzer measurements of an ion beam on the low potential side of a currentfree double-layer, IEEE Trans. Plasma Sci. 33, 336-337 (2005).

${ }^{6}$ C. Charles, R.W. Boswell, P. Alexander, C. Costa, and O. Sutherland, Helicon double layer thrusters, 42nd AIAA/ASME/SAE/ASEE Joint Propulsion Conference, AIAA 2006-4838 (2006), 4 pp.

${ }^{7}$ A.M. Keesee, E.E. Scime, C. Charles, A. Meige, and R. Boswell, The ion velocity distribution function in a current-free double layer, Phys. Plasmas 12, 093502-1 to -7 (2005).

${ }^{8}$ X. Sun, A..M. Keesee, C. Biloiu, E.E. Scime, A. Meige, C. Charles, and R.W. Boswell, Observations of ion-beam formation in a current-free double layer, Phys. Rev. Lett. 95, 025004-1 to -4 (2005).

${ }^{9}$ A. Meige, R.W. Boswell, C. Charles, J.P. Boeuf, G. Hagelaar, and M.M. Turner, One-dimensional simulation of an ion beam generated by a current-free double-layer, IEEE Trans. Plasma Sci 33 Part 1, 334-335 (2005).

${ }^{10}$ A. Meige, R.W. Boswell, C. Charles, and M.M. Turner, One-dimensional particle-in-cell simulation of a currentfree double layer in an expanding plasma, Phys. Plasmas 12, 052317-1 to -10 (2005).

${ }^{11}$ R. Walker, N. Plihon, P. Chabert, and J-L Raimbault, Experimental studies of helicon double layers for future high power plasma propulsion, 42nd AIAA/ASME/SAE/ASEE Joint Propulsion Conference, AIAA 2006-4844 (2006), 4 pp.

12 C. Charles, A review of recent laboratory double layer experiments, Plasma Sources Sci. Technol. 16, R1-R25 (2007).

${ }^{13}$ F.F. Chen, Physical mechanism of current-free double layers, Phys. Plasmas 13, 034502-1 to -3 (2006).

${ }^{14}$ O. Sutherland, C. Charles, N. Plihon, and R.W. Boswell, Experimental evidence of a double layer in a large volume helicon reactor, Phys. Rev. Lett. 95, 205002-1 to -4 (2005), Fig. 2.

${ }^{15}$ C. Charles, High source potential upstream of a current-free electric double layer, Phys. Plasmas 12, $044508-1$ to $-4(2005)$.

${ }^{16}$ F.N. Gesto, B.D. Blackwell, C. Charles, and R.W. Boswell, Ion detachment in the helicon double-layer thruster exhaust beam, J. Propulsion and Power 22, 24-30 (2006).

${ }^{17}$ F. R. Chang-Diaz, Research status of the Variable Specific Impulse Magnetoplasma Rocket, Trans. Fusion Technol. 35, 87-93 (1999).

${ }^{18}$ R. Winglee, T. Ziemba, L. Giersch, J. Prager, J. Carscadden, and B. R. Roberson, Simulation and laboratory validation of magnetic nozzle effects for the high power helicon thruster, Phys. Plasmas 14, 063501-1 to -14 (2007).

${ }^{19}$ F.F. Chen and H. Torreblanca, Large-area helicon plasma source with permanent magnets, Plasma Phys. Control. Fusion 49, A81-A93 (2007).

${ }^{20}$ F.F. Chen, Helicon plasma source with permanent magnets, U.S. Patent Application No. 60/693,054 (2005).

${ }^{21}$ J.P. Squire, F.R. Chang-Diaz, T.W. Glover, V.T. Jacobson, G.E. McCaskill, D.S. Winter, F.W. Baity, M.D. Carter, and R.H. Goulding, High power light gas helicon plasma source for VASIMR, Thin Solid Films 506507, 579-582 (2006). 
${ }^{22}$ M.D. Carter, F.W. Baity, Jr., G.C. Barber, R.H. Goulding, Y. Mori, D.O. Sparks, K.F. White, E.F. Jaeger, F.R. Chang-Diaz, and J.P. Squire, Comparing experiments with modeling for light ion helicon plasma sources, Phys. Plasmas 9, 5097-5110 (2002).

${ }^{23}$ T. Ziemba, P. Euripides, J. Slough, R. Winglee, L. Giersch, J. Carscadden, T. Schnackenberg and S. Isley, Plasma characteristics of a high power helicon discharge, Plasma Sources Sci. Technol. 15, 517-525 (2006).

${ }^{24}$ K. Sasaki, H. Kokubu, D. Hayashi, K. Kadota, Development of a compact nitrogen radical source by heliconwave discharge employing a permanent magnet, Thin Solid Films 386, 243-247(2001).

${ }^{25}$ K.P. Shamrai, Y.V. Virko, V.F. Virko, and A.I. Yakimendo, Compact helicon plasma source with permanent magnets for electric propulsion application, 42nd AIAA/ASME/SAE/ASEE Joint Propulsion Conference, 9-12 July 2006, Sacramento, CA, AIAA 2006-4845.

${ }^{26}$ F.F. Chen, The low-field density peak in helicon discharges, Phys. Plasmas 10, 2586-2592 (2003).

${ }^{27} \mathrm{~S}$. Cho, The resistance peak of helicon plasmas at low magnetic fields, Phys. Plasmas 13, 033504-1 to -7 (2006).

${ }^{28}$ F.F. Chen, Helicon Plasma Sources, in "High Density Plasma Sources", ed. by Oleg A. Popov (Noyes Publications, Park Ridge, NJ), Chap. 1 (1995).

${ }^{29}$ D. Arnush, The role of Trivelpiece-Gould waves in antenna coupling to helicon waves, Phys. Plasmas 7, 30423050 (2000).

${ }^{30} \mathrm{http}: / /$ www.ee.ucla.edu/ ltptl/presentations

${ }^{31}$ TG modes are electrostatic electron cyclotron waves confined to a cylinder. In a helicon discharge, they are excited by the image charges or currents of helicon waves when they bounce off the radial boundary. Numerous theoretical papers have shown that a major RF absorption mechanism in helicon discharges is mode conversion into TG waves and the strong damping of these waves as they propagate slowly inward from the boundary. TG waves have been detected experimentally in helicon discharges.

${ }^{32}$ K.P. Shamrai and V.B. Taranov, Resonance wave discharge and collisional energy absorption in helicon plasma source, Plasma Phys. Control. Fusion 36, 1719-1735 (1994).

${ }^{33}$ M. Nisoa, Y. Sakawa, and T. Shoji, Characterization of Plasma Production by $m=0$ Standing Helicon Waves, Jpn. J. Appl. Phys. 40, Pt. 1, 3396-3404 (2001).

${ }^{34}$ V.F. Virko, G.S. Kirichenko, and K.P. Shamrai, Geometrical resonances of helicon waves in an axially bounded plasma, Plasma Sources Sci. Technol. 11, 10-26 (2002).

${ }^{35}$ G.R. Tynan, A.D. Bailey III, G.A. Campbell, R. Charatan, A. de Chambrier, G. Gibson, D.J. Hemker, K. Jones, A. Kuthi, C. Lee, T. Shoji, and M. Wilcoxson, Characterization of an azimuthally symmetric helicon wave high density plasma source, J. Vac. Sci. Technol. A 15, 2885-2892 (1997).

${ }^{36}$ K.P. Shamrai, V.P. Pavlenko, and V.B. Taranov, Excitation, conversion and damping of waves in a helicon plasma source driven by an $m=0$ antenna, Plasma Phys. Control. Fusion 39, 505-530 (1997).

${ }^{37}$ Y. Sakawa, T. Takino, and T. Shoji, Control of antenna coupling in high-density plasma production by $m=0$ helicon waves, Appl. Phys. Lett. 73, 1643-1645 (1998).

${ }^{38}$ D.D. Blackwell, T.G. Madziwa, D. Arnush, and F.F. Chen, Evidence for Trivelpiece-Gould Modes in a Helicon Discharge, Phys. Rev. Lett. 88, 145002-1 to -4 (2002).

${ }^{39}$ F.F. Chen and H. Torreblanca, Density jump in helicon discharges, Plasma Sources Sci. Technol. 16, 593-596 (2007).

${ }^{40}$ H. Torreblanca, Multitube Helicon Source With Permanent Magnets, Thesis, UCLA (2008).

${ }^{41}$ F.F. Chen, J.D. Evans, and G.R. Tynan, Design and performance of distributed helicon sources, Plasma Sources Sci. Technol. 10, 236-249 (2001).

${ }^{42}$ F.F. Chen, Plasma ionization by helicon waves, Plasma Phys. Control. Fusion 33, 339-364 (1991).

${ }^{43}$ A. W. Trivelpiece and R.W. Gould, Space Charge Waves in Cylindrical Plasma Columns, J. Appl. Phys. 30, 1784-1793 (1959). 
${ }^{44}$ J.P. Rayner, A.D. Cheetham, and G.N. French, Radio frequency matching for helicon plasma sources, J. Vac. Sci. Technol. A 14, 2048-2055 (1996).

${ }^{45}$ V.A. Godyak, New ICP Technology for Semiconductor Material Processing, Presentation given at the Plasma Etch Users Group, Santa Clara, September 2003.

${ }^{46}$ Here italic $L$ is length while roman $L$ is inductance, and the optimized dimensions are used.

${ }^{47}$ M.A. Lieberman and A.J. Lichtenberg, Principles of Plasma Discharges and Materials Processing, 2nd ed. (Wiley-Interscience, 2005), Fig. 3.17.

${ }^{48}$ A. Simon, Phys. Rev. 98, 317-318 (1955).

${ }^{49}$ A. Fruchtman, Phys. Rev. Lett. 96, 065002-1 to -4 (2006). 